\title{
CGH-based microarray detection of cryptic and novel copy number alterations and balanced translocations in cytogenetically abnormal cases of b-cell all
}

\author{
Roger A. Schultz ${ }^{1^{*}}$, Karen Tsuchiya ${ }^{2}$, Aubry Furrow ${ }^{1}$, Marilyn L. Slovak ${ }^{3}$, Lisa D. McDaniel ${ }^{1}$, \\ Meaghan Wall ${ }^{4}$, Eric Crawford ${ }^{5}$, Yi Ning ${ }^{6}$, Reza Saleki ${ }^{7}$, Min Fang ${ }^{8}$, Victoria Cawich $^{1}$, \\ Caitlin E. Johnson ${ }^{1}$, Sara L. Minier ${ }^{1}$, Nicholas J. Neill ${ }^{9}$, S. Annie Morton ${ }^{1}$, Steve Byerly ${ }^{10}$, \\ Urvashi Surti $^{11}$, Theresa C. Brown ${ }^{12}$, Blake C. Ballif ${ }^{10}$, Lisa G. Shaffer ${ }^{10}$

\footnotetext{
${ }^{1}$ Signature Genomic Laboratories, PerkinElmer, Inc., Spokane, USA; ${ }^{*}$ Corresponding Author: roger.schultz@perkinelmer.com

${ }^{2}$ Seattle Children's Hospital, Seattle, USA

${ }^{3}$ Sonora Quest Laboratories, Tempe, USA

${ }^{4}$ Victorian Cancer Cytogenetics Service, St. Vincent's Hospital Melbourne, Fitzroy, Australia

${ }^{5}$ Genetics Associates, Inc., Nashville, USA

${ }^{6}$ Johns Hopkins University School of Medicine, Baltimore, USA

${ }^{7}$ Pathology Associates Medical Laboratories (PAML), Spokane, USA

${ }^{8}$ Fred Hutchinson Cancer Research Center, Seattle, USA

${ }^{9}$ Department of Molecular and Human Genetics, Baylor College of Medicine, Houston, USA

${ }^{10}$ Paw Print Genetics, Genetic Veterinary Sciences, Inc., Spokane, USA

${ }^{11}$ Magee-Womens Hospital, University of Pittsburgh Medical Center, Pittsburgh, USA

${ }^{12}$ Empire Genomics, Buffalo, USA
}

Received 14 March 2013; revised 2 April 2013; accepted 15 May 2013

Copyright (C) 2013 Roger A. Schultz et al. This is an open access article distributed under the Creative Commons Attribution License, which permits unrestricted use, distribution, and reproduction in any medium, provided the original work is properly cited.

\section{ABSTRACT}

Acute lymphoblastic leukemia (ALL) is the most common malignancy in children, with the majority of cases being of precursor B-cell phenoltype. Conventional cytogenetic analysis plays an important role in the diagnosis of B-cell ALL, identifying characteristic chromosomal abnormalities associated with a given prognosis therein facilitating optimized treatment. The more recent introduction of microarray technology to the analysis of B-cell ALL has afforded both higher resolution for the detection of known abnormalities and an ability to identify novel copy number abnormalities (CNAs) with potential clinical relevance. In the current study, microarray analysis was performed on 20 cytogenetically abnormal B-cell ALL cases (10 pediatric and 10 adult), while a novel microarray-based balancedtranslocation detection methodology (translocation CGH or tCGH) was applied to that subset of cases with a known or suspected recurrent balanced translocation. Standard microarray analysis identified that CNAs was not detected by previous conventional cytogenetics in $75 \%(15 / 20)$ cases. tCGH identified 9/9 (100\%) balanced translocations defining BCR/ABL1 (x4), ETV6/RUNX1 (x3), and MLL/AFF1 (x2) breakpoints with high resolution. The results illustrate the improved molecular detail afforded by these technologies and a comparison of translocation breakpoints, CNAs and patient age offers new insights into tumor biology with potential prognostic significance.

Keywords: Acute Lymphoblastic Leukemia; B-Cell ALL; Microarray; Balanced Translocation; Translocation CGH; Hematologic Malignancies

\section{INTRODUCTION}

B-cell acute lymphoblastic leukemia (B-cell ALL) is the most common pediatric malignancy. Despite significant advances in treatment resulting in a cure rate of nearly $80 \%$ in the pediatric population [1-3], approximately $20 \%$ to $25 \%$ of children and more than half of adult patients experience relapse $[4,5]$. Conventional cytogenetic analysis has been an integral part of the evaluation of B-cell ALL, influencing prognosis and treatment decisions [6,7]. Specific cytogenetic findings, such as the $t$ $(9 ; 22)$ (q34;q11.2) resulting in $B C R / A B L 1$ fusion, $M L L$ 
rearrangements, and hypodiploidy are associated with a poor prognosis [8]. In contrast, hyperdiploidy, particularly involving gains of chromosomes 4 and 10 , and the $\mathrm{t}$ $(12 ; 21)$ (p13;q22) resulting in ETV6/RUNX1 fusion, are associated with a good prognosis [8]. However, some cases of B-cell ALL lack alterations detectable by conventional cytogenetics and, importantly, some patients with favorable cytogenetic alterations still relapse, which may indicate that that there are additional undetected chromosome aberrations [9].

In recent years, genomic profiling of B-cell ALL using newer technologies has uncovered additional genetic alterations that cannot be detected by conventional cytogenetics [10-12]. Common alterations include genes involved in the regulation of B-cell development and differentiation (PAX5, IKZF1, EBF1, LEF1 and TCF3), cell cycle regulation and tumor suppression (CDKN2A, RB1 and PTEN), and lymphoid signaling (CD200, BTLA, and $C R L F 2$ ) [10-16]. Many of these alterations are submitcroscopic deletions that can be identified using highdensity oligonucleotide comparative genomic hybridization (CGH)-based microarrays (aCGH) and single nucleotide polymorphism (SNP) microarrays. These novel findings have not only led to insights concerning the boilogy of B-cell ALL, but some have also been shown to be predictors of high-risk disease and have potential as future therapeutic targets. For example, deletions of IKZF1, often too small to be detected by fluorescence in situ hybridization (FISH), have been found to be a strong predictor of relapse $[13,17]$. Thus, it will be important in the clinical laboratory to employ technology with the capacity to identify these submicroscopic alterations that are below the resolution level of conventional cytogenetics.

Microarray-based detection of copy number alterations (CNAs) has become standard of care for the diagnosis of most constitutional chromosomal imbalances in children with developmental disabilities [18], but its potential for the evaluation of neoplasia in the clinical laboratory has been compromised by the inability to detect balanced rearrangements, which are important recurrent diagnostic and prognostic markers. To circumvent the limitation of balanced translocation detection, traditional microarray may be supplemented with routine karyotyping, selected FISH studies, targeted rtPCR analysis when appropriate, or a broader and more recently described technique of translocation array (tCGH)] [19,20]. This novel technique couples linear amplification of genomic DNA using multiplexed primer sets targeted to a set of translocation breakpoint intervals with microarray analysis, permitting diagnostic detection of balanced translocations with unprecedented genomic resolution of the breakpoints. In the study presented here we characterize 20 cases of cytogenetically abnormal B-cell ALL by micro- array analysis, including microarray analysis of putative translocations in 10 cases using a single tCGH assay designed to interrogate genomic DNA for the presence of 8 distinct translocations common in B-cell ALL. Joint analysis of aCGH and tCGH results provides detailed resolution of CNAs and genomic breakpoints offering potential new insights into tumor biology and prognostic significance.

\section{MATERIALS AND METHODS}

\subsection{Specimen Ascertainment}

Residual bone marrow or leukemic peripheral blood was obtained after routine testing from seven clinical cytogenetic laboratories for DNA extraction. Routine Gbanded chromosome analysis was performed in the referring laboratories on all samples, and many cases were also analyzed by FISH as directed by standard clinical practice. The cases consisted of 13 bone marrow and four leukemic peripheral blood specimens at the time of initial diagnosis, one bone marrow three weeks after initial diagnosis, and two bone marrow specimens at relapse. Ten cases were from pediatric patients ( 1 - 18 years old), and ten cases were from adults (25 - 73 years old). Samples were de-identified for all information other than B-cell ALL indication for study, age, sex and prior cytogenetic findings. Except as otherwise indicated, additional specimen was not available for any post-analysis confirmatory studies. This study was conducted in accordance to Signature Genomics' Spokane-IRB approved protocol.

\subsection{DNA Extraction}

Genomic DNA was extracted from unenriched blood and bone marrow specimens using the Gentra Puregene Blood Kit (Qiagen, Germantown, MD) according to the manufacturer's instructions. Two million cells or $150 \mu \mathrm{l}$ (if cell counts were unavailable) of blood or bone marrow were used as starting material. Additional Cell Lysis Solution (Gentra Puregene Blood Kit) was added to samples with high viscosity to ensure complete lysis. Samples were stabilized in Cell Lysis Solution within 24 to 48 hours of specimen receipt, when possible, to ensure high-quality DNA for use on the microarray.

DNA quality was assessed by measuring DNA concentration, 260/280 $\mathrm{nm}$ and 260/230 nm readings on a NanoDrop 2000 Spectrophotometer (Thermo Scientific, Waltham, MA). The DNA was also run on a $1 \%$ agarose gel with ethidium bromide to detect degradation. To be included in the study, samples had to have minimal degradation with $260 / 280 \mathrm{~nm}$ values near 1.8 and 260/230 $\mathrm{nm}$ readings greater than 1.35 . 


\subsection{Oligonucleotide Microarray Labeling Hybridization and Analysis}

Array CGH for copy number analysis was performed using a 135K-feature whole-genome oligonucleotide microarray (aCGH) (Signature OncoChip ${ }^{\circledR}$, designed by Signature Genomics, Spokane, WA; manufactured by Roche NimbleGen, Madison, WI). When compared to probe coverage over the rest of the genome, this microarray has denser oligonucleotide coverage over 1893 cancer features, including genes with known roles in hematologic malignancies or solid tumors in which deletions or mutations had been previously reported, genes with suspected roles in cancer based on prior expression studies without specific evidence of genomic copy changes, genes with previously speculated roles based solely on association with a biological pathway or gene family, and genes involved in protein and microRNA (miRNA) coding. The microarray has an average coverage of one oligonucleotide per $0.2-7.0 \mathrm{~kb}$ for the targeted cancer features and genomic backbone coverage of one oligonucleotide per $35 \mathrm{~kb}$. Labeling, hybridization, and washing were performed using previously published methods [21]. Data were analyzed and displayed using custom oligonucleotide aCGH data analysis and visualization software (Oncoglyphix ${ }^{\circledR}$; Signature Genomics) as previously described [22]. Variants were identified based on the number of consecutive oligonucleotides involved and the magnitude in shift for the $\log 2$ ratio, with 5 - 99 oligonucleotides requiring a shift $\geq 0.300,100-999$ a shift $\geq 0.200$, and $\mathrm{a} \geq 1000$ a shift $\geq 0.100$.

\subsection{Translocation $\mathrm{CGH}$}

For 10 of the 20 cases, prior chromosome and/or FISH analysis revealed evidence of a clinically relevant balanced translocation. For eight of those cases, translocations were identified by karyotypes only, for one case by both FISH and karyotype, and for one case by FISH only. Nine of those 10 cases had sufficient DNA to be assessed by both aCGH and the translocation CGH array (tCGH). For one additional case (Case 10), aCGH revealed a breakpoint for a genomic copy gain within the MLL gene, raising suspicion of a putative translocation. Thus, case 10 was also evaluated by the tCGH technique, a microarray strategy is based on linear amplification of the junction between partner genes involved in balanced translocations $[19,20]$. For tCGH, cases were analyzed using a single multiplexed assay designed to detect eight possible translocations (Table 1) that are all recurrent in B-cell

Table 1. Primer coverage in the multiplex tCGH assay designed to simultaneously detect eight different balanced translocations associated with B-cell ALL.

\begin{tabular}{|c|c|c|c|c|c|c|c|c|c|c|}
\hline \multicolumn{2}{|c|}{ Translocation } & \multicolumn{4}{|c|}{ Array 1 primer pool } & \multicolumn{4}{|c|}{ Array 2 primer pool } & \multirow{2}{*}{$\begin{array}{l}\text { Conditions and } \\
\text { frequency }\end{array}$} \\
\hline $\begin{array}{l}\text { Gene } \\
\text { fusion }\end{array}$ & Rearrangement & Gene & Strand & $\begin{array}{l}\text { No. of } \\
\text { primers }\end{array}$ & $\begin{array}{c}\text { Mean } \\
\text { spacing }(\mathrm{kb})\end{array}$ & Gene & Strand & $\begin{array}{l}\text { No. of } \\
\text { primers }\end{array}$ & $\begin{array}{c}\text { Mean } \\
\text { spacing }(\mathrm{kb})\end{array}$ & \\
\hline$B C R / A B L 1$ & $\mathrm{t}(9 ; 22)(\mathrm{q} 34 ; \mathrm{q} 11.2)$ & $A B L 1$ & - & 47 & 3.3 & $B C R$ & + & 55 & 2.5 & $\begin{array}{c}5 \% \text { of pediatric and } 30 \% \text { of } \\
\text { adult ALL, } 1 \% \text { of AML, and } \\
\text { nearly } 100 \% \text { of CML with only } \\
95 \% \text { observed by } \\
\text { cytogenetics }[48]\end{array}$ \\
\hline$M L L / A F F 1$ & $\mathrm{t}(4 ; 11)(\mathrm{q} 21 ; \mathrm{q} 23)$ & $A F F 1$ & - & 35 & 2.9 & $M L L$ & + & 11 & 2.1 & $\begin{array}{l}2 \% \text { of childhood ALL [49], } \\
<1 \% \text { of AML [50-52], 3\% of } \\
\text { t }(4 ; 11) \text { seen in AML }[53]\end{array}$ \\
\hline$M L L / M L L T 3$ & $\mathrm{t}(9 ; 11)(\mathrm{p} 22 ; \mathrm{q} 23)$ & MLLT3 & + & 30 & 3.2 & $M L L$ & + & 11 & 2.1 & $\begin{array}{l}\text { Reported rarely in ALL [54], } \\
7 \% \text { of de novo AML, } 46 \% \text { of } \\
\text { secondary AML, seen } \\
\text { exclusively in M5 in de novo } \\
\text { cases, M0 and M4 seen in } \\
\text { secondary cases [55] }\end{array}$ \\
\hline$P B X 1 / T C F 3$ & $\mathrm{t}(1 ; 19)(\mathrm{q} 23 ; \mathrm{p} 13)$ & TCF3 & - & 7 & 2.4 & $P B X 1$ & - & 41 & 3.2 & $\begin{array}{l}5 \% \text { of childhood ALL, } 20 \% \text { of } \\
\text { childhood pre B-cell ALL, also } \\
\text { seen in T-ALL and AML [56] }\end{array}$ \\
\hline ETV6/RUNX1 & $\mathrm{t}(12 ; 21)(\mathrm{p} 13 ; \mathrm{q} 22)$ & $R U N X 1$ & + & 54 & 3.2 & ETV6 & + & 83 & 3.0 & $\begin{array}{l}25 \% \text { of pediatric B-cell ALL, } \\
3 \% \text { of adult B-cell ALL [48] }\end{array}$ \\
\hline$M L L / E P S 15$ & $\mathrm{t}(1 ; 11)(\mathrm{p} 32 ; \mathrm{q} 23)$ & EPS15 & + & 27 & 3.2 & $M L L$ & + & 11 & 2.1 & $\begin{array}{l}\text { Reported rarely in ALL [54], } \\
<1 \% \text { of AML [50] }\end{array}$ \\
\hline$H L F / T C F 3$ & $\mathrm{t}(17 ; 19)(\mathrm{q} 22 ; \mathrm{p} 13)$ & TCF3 & - & 7 & 2.4 & $H L F$ & - & 6 & 2.4 & $\begin{array}{c}1 \% \text { of pediatric B-cell ALL } \\
{[56,57]}\end{array}$ \\
\hline$M L L / M L L T 1$ & $\mathrm{t}(11 ; 19)(\mathrm{q} 23 ; \mathrm{p} 13.3)$ & $M L L T 1$ & + & 23 & 3.1 & $M L L$ & + & 11 & 2.1 & $\begin{array}{l}2 \% \text { of childhood AML }[51], \\
\text { seen frequently in ALL }[54,58]\end{array}$ \\
\hline To & tal primers & & & 223 & & & & 196 & & \\
\hline
\end{tabular}


ALL. For each specimen tested, two separate linear amplification reactions were performed using multiplex primer pools designed to amplify one partner of each of the eight targeted balanced translocations (Table 1).

Unique DNA primers for genes of interest were designed using MacVector V11.1 (MacVector, Inc., Cary, NC) software with an average spacing of one primer every 2000 to $3500 \mathrm{bp}$. Primers were mixed into genespecific and multiplexed reactions at a final concentration of $200 \mathrm{nM}$ for each primer. Linear amplification reactions using $600 \mathrm{ng}$ genomic DNA were performed using the FailSafe PCR System with PreMix Choice using a single buffer (FailSafe PCR 2X PreMix D, Epicentre, Madison, WI) as described by the manufacturer, with 5\% DMSO and a final reaction volume of $50 \mu \mathrm{l}$. After an initial denaturation at $94^{\circ} \mathrm{C}$ for 2 minutes, reactions were amplified for 10 cycles with denaturation at $94^{\circ} \mathrm{C}$ for 10 seconds, annealing at $62^{\circ} \mathrm{C}$ for 30 seconds, and elongation at $68^{\circ} \mathrm{C}$ for 20 minutes. This was followed by another 10 cycles with the same conditions except the denaturation time was increased to 15 seconds and the elongation time was extended by an additional 20 seconds on each successive cycle. The QuickStep 2 PCR Purification Kit (EdgeBio, Gaithersburg, MD) was used according to manufacturer's specifications to purify samples after the linear amplification reactions and prior to labeling.

Microarray analysis was performed using the following methods. Amplified DNA ( $40 \mu 1$ of the purified, linear amplification product) and $600 \mathrm{ng}$ of non-amplified, genomic control DNA were labeled with Cyanine dyes (Cy5 for the specimen and Cy3 for the control) using the NimbleGen Dual-Color DNA Labeling Kit (Roche NimbleGen) and purified as described by the manufacturer. Labeled specimen and control DNA were quantified using the NanoDrop 8000 Spectrophotometer (Thermo Scientific), combined (31 $\mu \mathrm{g}$ each), and coprecipitated with $50 \mu \mathrm{g}$ of Human Cot-1 DNA (Invitrogen, Carlsbad, CA) in isopropanol. Co-precipitated DNAs were hybridized to the arrays at $42^{\circ} \mathrm{C}$ for $40-72$ hours and then washed as described by the manufacturer (Roche NimbleGen). Arrays were scanned at $2 \mu \mathrm{m}$ using a Roche NimbleGen MS 200 Microarray Scanner and the data were analyzed with NimbleScan 2.6 software. Results were displayed and analyzed with Oncoglyphix ${ }^{\circledR}$.

\subsection{Affymetrix SNP 6.0 Array Analysis}

For Case 2, SNP microarray analysis was performed using the Affymetrix SNP Array 6.0 (Affymetrix, Santa Clara, CA) according to manufacturer's instructions. Data were analyzed using Affymetrix Chromosome Analysis Suite (ChAS) software also in accordance with the manufacturer's instructions and visualized with Oncoglyphix ${ }^{\circledR}$.

\section{RESULTS}

\subsection{Detection of Balanced Translocations by TCGH}

Prior chromosome analysis and/or FISH indicated the presence of a clinically relevant balanced translocation in 10 of 20 cases (Tables 2 and 3 ) and aCGH results identified a potential additional case (case 10) with a CNA within the MLL gene indicative of a possible translocation. Of the 11 cases of interest, 10 had sufficient DNA to perform the tCGH assay (Table 3). Of these, all nine cases that revealed evidence of a translocation by karyotypes or FISH analysis demonstrate translocations by tCGH. These included four cases of $t(9 ; 22)$, three cases of $\mathrm{t}(12 ; 21)$, and two cases of $\mathrm{t}(4 ; 11)$ (Figure 1). Case 10 , which revealed a breakpoint for a genomic copy gain by aCGH, did not exhibit any translocation when analyzed with the tCGH assay. Results provided high resolution mapping of the breakpoints, with precise definition dependent on the oligonucleotide coverage present on the tCGH arrays (Figure 1, Table 3). All translocation breakpoints were confirmed by PCR using individual primers flanking breakpoints defined by tCGH and standard PCR techniques (data not shown).

\subsection{Detection of CNAs}

In 15 of the 20 cases (75\%), CNAs that were not identified by G-banded analysis or FISH were detected by microarray (Table 2). These alterations ranged from focal intragenic imbalances to gains or losses of many megabases of DNA. Focal CNAs, predominantly deletions, involving genes that have previously been described in ALL included PAX5 (6 cases), BTG1 (4 cases), IKZF1 (6 cases), TOX (3 cases), EBF1 (2 cases), LEF1 (1 case), BTLA (1 case), NR3C1 (1 case), FOXP1 (1 case), and TBL1XR1 (1 case). Of the six cases with PAX5 CNAs, four consisted of heterozygous deletions ( $\sim 200$ to $250 \mathrm{~kb}$ ) involving one or more exons at the 5 ' end of the gene (Cases 6, 9, 12, and 19; Table 2 and Figure 2(a)), one case showed both a focal intronic loss and a multicopy gain involving exons 2 - 5 in PAX5 (Case 13; Table 2 and Figure 2(b)) and another case demonstrated amplification within PAX5 that included exons 2 - 5 (Case 1, Table 2 and Figure 2(b)). In addition to the six cases with focal PAX5 CNAs, one additional case (Case 5; Table 2) had heterozygous loss of PAX5 as the result of loss of $9 \mathrm{p}$ from a dic $(9 ; 20)$. Note that for TBL1XR1, the single observed deletion (Case 8 ) ends $37 \mathrm{~kb}$ distal to the 5 '-end of the gene and may not impact expression.

All four cases with t $(9 ; 22)$ had loss of IKZF1 including deletions of varying sizes, and one $\mathrm{t}(9 ; 22)$ positive case had monosomy 7 (Case 3). One case showed biallelic loss involving IKZF1 due to loss of $7 p$ from an i (7q), 
Table 2. Summary of microarray and other cytogenetic results for 20 B-cell ALL cases.

\begin{tabular}{|c|c|c|c|c|c|c|}
\hline Case & Age & $\begin{array}{l}\text { Cytogenetic } \\
\text { Results }\end{array}$ & $\begin{array}{l}\text { FISH } \\
\text { Results }\end{array}$ & Microarray (hg18) & $\begin{array}{c}\text { Significant } \\
\text { microarray findings }\end{array}$ & $\begin{array}{c}\text { Unclear } \\
\text { microarray findings }\end{array}$ \\
\hline 1 & 18 & $\begin{array}{c}\text { 47, XY, add (3) } \\
\text { (p?12), +5, ? add } \\
\text { (5) (q?31), add (7) } \\
\text { (q32), add (9) } \\
\text { (p?13), } \\
\text { der (9) t (3;9) } \\
\text { (p21; p?21), add } \\
\text { (11) (q?23) [16]/46, } \\
\text { XY [6] }\end{array}$ & $\begin{array}{l}\text { nuc ish (CDKN2Ax0, } \\
\text { CEP9x2) [15/200], } \\
\text { (MLLx2) [200] }\end{array}$ & 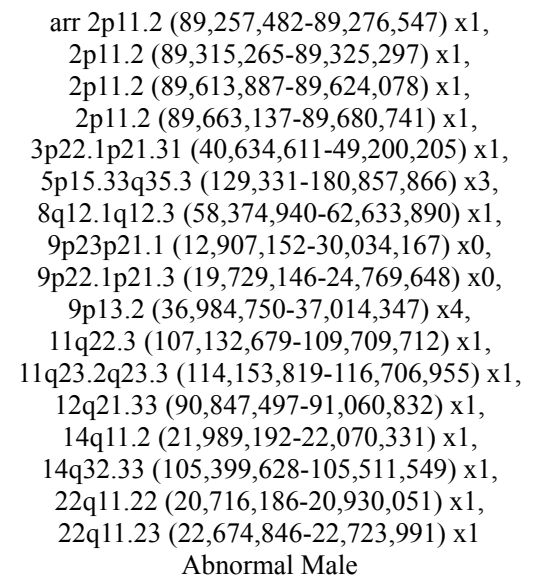 & $\begin{array}{l}\text { Trisomy } 5 \\
9 \mathrm{p} \text { deletion with } \\
\text { biallelic deletion of } \\
C D K N 2 A \text { and } \\
\text { CDKN2B } \\
\text { Deletion of ATM } \\
\text { Intragenic PAX5 } \\
\text { amplification }\end{array}$ & $\begin{array}{c}\text { IGK, IGH, GSTT1 } \\
\text { deletions } \\
\text { 3p deletion including } \\
\text { several genes } \\
\text { (CTNNB1 involved in } \\
\text { wnt-catenin } \\
\text { signaling) }\end{array}$ \\
\hline 2 & 65 & $\begin{array}{c}\text { 46, XX, add (4) } \\
\text { (p11.2) } \\
{[4] / 46, X X[16]}\end{array}$ & NA & 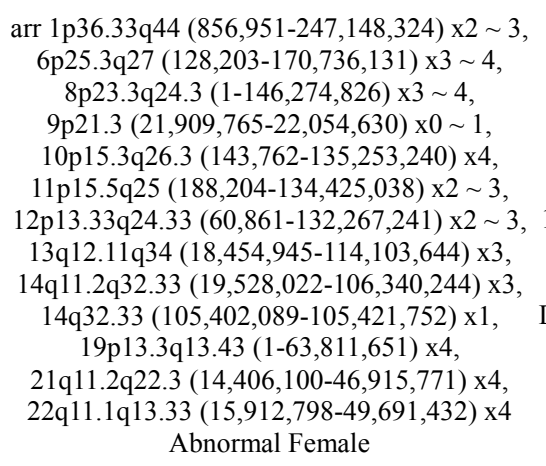 & $\begin{array}{l}\text { Mosaic or } \\
\text { non-mosaic } \\
\text { tetrasomy for } \\
1,6,8,10,11,12,13,14, \\
19,21,22 \\
\text { Deletion of } C D K N 2 A \\
\text { and } C D K N 2 B\end{array}$ & Deletion in $I G H$ \\
\hline 3 & 65 & $\begin{array}{c}45, \mathrm{XX},-7, \mathrm{t}(9 ; 22) \\
(\mathrm{q} 34 ; \mathrm{q} 11.2) \\
{[12] / 46, \mathrm{XX}[8]}\end{array}$ & NA & $\begin{array}{c}\text { arr 1p33 }(51,174,821-51,208,318) \times 1, \\
\text { 2p11.2 }(88,696,337-89,376,097) \times 1, \\
2 \mathrm{p} 11.2(89,561,572-89,912,623) \times 1, \\
7 \mathrm{p} 22.3 \mathrm{q} 36.3(1-158,821,424) \times 1, \\
14 \mathrm{q} 32.33(105,401,418-105,602,516) \times 1, \\
22 \mathrm{q} 11.22(20,634,021-20,930,168) \times 1 \\
\text { Abnormal female }\end{array}$ & $\begin{array}{c}\text { Monosomy } 7 \\
(I K Z F 1)\end{array}$ & $\begin{array}{l}\text { Deletion involving } \\
\qquad D K N 2 C \\
\text { Ig deletions }\end{array}$ \\
\hline 4 & 73 & $\begin{array}{c}45, \mathrm{XX}, \operatorname{der}(3 ; 7) \\
(\mathrm{p} 10 ; \mathrm{q} 10) \\
{[6] / 46, \mathrm{XX}[14]}\end{array}$ & NA & $\begin{array}{c}\text { arr Xq27.3q28 }(144,670,466-154,876,029) \times 3, \\
\text { 2p11.2 }(89,014,482-89,348,801) \times 1, \\
\text { 2p11.2 }(89,590,599-89,912,623) \times 1, \\
\text { 3q11.2q21.1 (95,167,813-124,286,166)x1, } \\
\text { 3q21.3q26.2 }(130,654,972-169,688,965) \times 1, \\
\text { 7p22.3p11.1 }(130,978-57,515,054) \times 1, \\
\text { 7q31.33q36.3 }(124,339,888-158,804,322) \times 1, \\
\text { 9p21.3 }(21,005,360-22,356,302) \times 1, \\
\text { 12p13.31p12.3 }(9,155,209-17,104,302) \times 1, \\
\text { 14q32.33 }(105,345,270-105,612,992) \times 1, \\
\text { 22q11.22 }(20,643,480-20,930,051) \times 1 \\
\text { Abnormal female }\end{array}$ & $\begin{array}{l}\text { Large deletions in- } \\
\quad \text { volving } 3 \mathrm{q} \\
\text { Deletions of } 7 \mathrm{p} \\
\text { (IKZF1) and } 7 \mathrm{qter} \\
\text { Deletion of } C D K N 2 A \\
\quad \text { and } C D K N 2 B \\
\text { Gain of Xqter }\end{array}$ & $\begin{array}{c}\text { Ig deletions } \\
\text { Large 3q deletion } \\
\text { includes } E P H A 6, \\
\text { LNP1, TFG, ALCAM, } \\
\text { CBLB, CD200, BTLA, } \\
D R D 3, \text { ZBTB20, } \\
\text { GAP43, GSK3B, } \\
\text { MIR198, HSPBAP } 1, \\
\text { DIRC2 }\end{array}$ \\
\hline 5 & 1 & $\begin{array}{c}45, \mathrm{XY}, \operatorname{dic}(9 ; 20) \\
\text { (p13.2; q11.2) } \\
{[15] / 46, \mathrm{XY}[5]}\end{array}$ & $\begin{array}{c}\text { nuc ish (CDKN2Ax0, } \\
\text { CEP9x2) } \\
\text { [39/200], (CEP4, } \\
\text { ABL1, CEP10, MLL, } \\
\text { ETV6,D17Z1, } \\
\text { BCR) x2 [200] }\end{array}$ & $\begin{array}{c}\text { arr 2p11.2 }(88,932,826-89,325,297) \times 0 \sim 1, \\
\text { 2p11.2 }(89,613,887-89,912,623) \times 0 \sim 1, \\
9 p 24.3 p 13.2(188,160-36,846,193) \times 1 \sim 2, \\
9 p 21.3(21,132,703-22,868,081) \times 0,14 q 11.2 \\
(21,937,493-22,074,385) \times 1,14 \mathrm{q} 32.33 \\
(105,401,418-105,597,823) \times 120 \mathrm{q} 11.21 \mathrm{q} 13.33 \\
(30,646,941-62,359,694) \times 1 \text { Abnormal female }\end{array}$ & $\begin{array}{l}9 \mathrm{p} \text { deletion with } \\
\text { biallelic deletion of } \\
C D K N 2 A \text { and } \\
C D K N 2 B \\
\text { Large deletion } \\
\text { involving most of } \\
20 \mathrm{q}\end{array}$ & $\begin{array}{l}I G K \text { and } I G H \\
\text { deletions }\end{array}$ \\
\hline
\end{tabular}




\section{Continued}

\author{
46, XY, del (1) \\ (p34p36.1), del (6) \\ (q13q21), del (9) \\ (q13q22), add (10) \\ (p11.2), t (12;15) \\ (p13;q11.2), add (20) \\ (q11.2) [8]/46,sl, del (1) \\ (q32q42), t (6;21) \\ (p21;q22) [cp7]/46, \\ $\mathrm{XY}[5]$
}

46, XX, del (12) (p11.2p13), der (12) (12pter- $>12$ p13::21q22-

$73>21 \mathrm{q} 11.2:: 12 \mathrm{p} 13->$ 12qter), der (21) (21pter$>21 \mathrm{q} 11.2:: 12 \mathrm{p} 13:: 21 \mathrm{q} 22$ - > 21qter) [4]/46, X [18]

46, XY, der (12) (21pter$>1 \mathrm{p} 12:: 12 \mathrm{p} 13->$

12qter),der(19) (19pter->

$8 \quad 3 \quad 19 \mathrm{q} 13.1:: 12 \mathrm{p} 13:: 21 \mathrm{q} 22$ $->21$ qter $)$ $\operatorname{der}(21)(19 \mathrm{qter}->19 \mathrm{q} 13.1$ : :21p12-> 21q22::12p13-> 12pter)[8]/46, XY[12]

46, XX, t (9;22) (q34; 952 q11.2), add (20) (q11.2), $\operatorname{del}(21)$ (q21q22.3) [19]

48, XY, $+19,+$ mar $1052 \quad[1] / 51$, idem, +6 , $+13,+21[18] / 46$, $\mathrm{XY}[1]$ arr Xp21.1 (32,857,314-33,252,699) x0, $1 \mathrm{p} 36.32 \mathrm{p} 35.1(4,617,997-33,430,623) \times 1$,

1q42.13q42.2 (226,166,028-231,338,987) x1, 2 p11.2 $(88,814,253-89,348,801) \times 1$, 2 p11.2 $(89,590,599-89,912,416) \times 1$, 4q21.3q22.1 (88,176,109-88,211,992) x1, $5 q 31.3(142,709,231-142,776,040) \times 1$, $5 \mathrm{q} 35.1(170,669,914-170,671,184) \times 1$, 6p22.2p22.1 $(25,011,637-26,167,410) \times 1$, 6q16.3q21 $(104,163,415-112,301,878) \times 1$, 6q22.31 $(125,873,889-126,139,853) \times 1$,

NA 6q23.3q25.1 (137,784,022-151,883,174) x1, 9 p21.3p21.2 $(20,101,054-26,709,187) \times 1$, 9p21.3 $(21,492,342-22,054,630) \times 0$, $9 \mathrm{p} 13.2(37,018,557-37,223,691) \times 1$, 9q22.2q33.2 (91,719,661-123,306,663) x1, $14 \mathrm{q} 11.2(21,943,554-22,052,134) \times 1$, $14 \mathrm{q} 32.33(105,401,418-106,005,780) \times 1$, $20 \mathrm{p} 12.2(10,369,721-10,400,575) \times 1$, $21 \mathrm{q} 22.3(41,576,548-42,780,374) \times 1$, $22 \mathrm{q} 11.22(20,716,186-20,852,537) \times 1$ Abnormal male

arr 2p11.2 $(88,932,826-89,294,676) \times 1$, 2 p11.2 $(89,653,265-89,740,401) \times 1$, $3 q 26.2(170,532,557-170,862,897) \times 1$, $7 \mathrm{q} 34(141,848,384-142,230,606) \times 1$, NA $12 p 13.31 \mathrm{p} 11.1(7,183,961-34,107,615) \times 1$, $14 \mathrm{q} 32.33(105,402,089-105,592,918) \times 1$, 22q11.22 (21,570,725-21,577,402) x1 Abnormal female

arr 2p11.2 (88,932,826-89,912,901) x1, $3 q 26.32(178,434,828-179,384,704) \times 0$, 12p13.2 $(11,789,624-11,810,529) \times 1$,

NA 12p13.2p12.1 $(11,920,600-21,600,799) \times 1$, $14 q 11.2(21,978,139-22,054,108) \times 1$, $14 \mathrm{q} 32.33(105,402,089-105,588,340) \times 1$ Abnormal male

arr 2p11.2 (88,966,582-89,912,901) x1, $3 p 14.1(71,174,477-71,536,728) \times 1$, $3 q 13.2(113,612,958-113,698,834) \times 1$, 4q25 $(109,187,792-109,298,195) \times 1$, 6q21 $(109,346,113-109,434,527) \times 1$, $7 \mathrm{p} 12.2(50,214,532-50,444,361) \times 1$, 9p21.3 $(21,854,404-22,229,673) \times 1$, 9p13.2 $(37,003,771-37,259,921) \times 1$, $14 \mathrm{q} 11.2(21,978,139-22,078,275) \times 1$, $14 \mathrm{q} 32.33(105,346,465-105,550,411) \times 1$, $14 \mathrm{q} 32.33(105,780,835-106,261,145) \times 1$, $15 \mathrm{q} 21.3(55,091,960-55,138,547) \times 1$, $17 \mathrm{q} 21.1(35,496,033-35,597,274) \times 1$, 20q11.22q13.32 $(33,293,965-56,987,158) \times 1$, $22 \mathrm{q} 11.22(20,643,540-21,094,301) \times 1$ Abnormal female

arr 3q27.3 $(188,938,761-188,946,429) \times 1$, 6p25.3q27 $(1-170,899,992) \times 3$

$8 \mathrm{q} 24.21(128,815,237-128,819,784) \times 1$, $11 \mathrm{q} 14.2 \mathrm{q} 14.3(85,873,769-89,596,519) \times 4$,

$11 \mathrm{q} 22.1 \mathrm{q} 22.3(97,609,686-108,855,018) \times 4$, $11 \mathrm{q} 23.3(116,666,706-117,858,516) \times 4$,

NA 11q23.3q24.1 $(119,339,943-122,322,820) \times 4$, Trisomy $6,13,19,21$

$11 \mathrm{q} 24.2 \mathrm{q} 25(125,708,527-134,425,038) \times 4$,

$13 \mathrm{q} 12.11 \mathrm{q} 34(18,454,945-114,103,644) \times 3$, 19p13.3q13.43 $(1-63,811,651) \times 3$,

21q11.2q22.3 $(14,406,100-46,915,771) \times 3$, $22 q 11.23(21,888,154-21,893,301) \times 1$

ETV6 deletion
DMD deletion

Deletion of: $A F F 1$,

Ig and TCR deletions

NR3C1, TLX3, PAX5, 1p deletion with large C20orf94 number of cancer features

Biallelic deletion of CDKN2A

1q deletion (WNT3A and EGLN1)

4q deletion $(A F F 1)$

MECOM deletion

ETV6 deletion

Ig and TCR deletions

Biallelic deletion

abutting 5 'end of TBL1XR1

IGK and IGH deletions

TRA/D deletion

Deletions involving: Deletions of: $L E F 1$, FOXP1, BTLA, SESN1, TCF12 and IKZF $1, C D K N 2 A / B, \quad C A S C 3$ and $P A X 5$

IGH, IGL and Large deletion of $20 \mathrm{q} \quad T C R A / D$ deletions Abnormal male 


$$
\begin{aligned}
& \text { 42, XY, del (3) (p13), del } \\
& \text { (4) (q21), add (5) } \\
& \text { (q10), -7, -9, -10, del (11) } \\
& \begin{array}{ll}
11 \quad 66 \quad \text { (q24), + der (11) } \\
\quad \text { ? dic } \mathrm{r}(11 ; 11)
\end{array} \\
& \text { (q10;q10) trp qdp (11) } \\
& \text { (q23q23), -16,-17 } \\
& \text { [10]/46, XY[5] }
\end{aligned}
$$

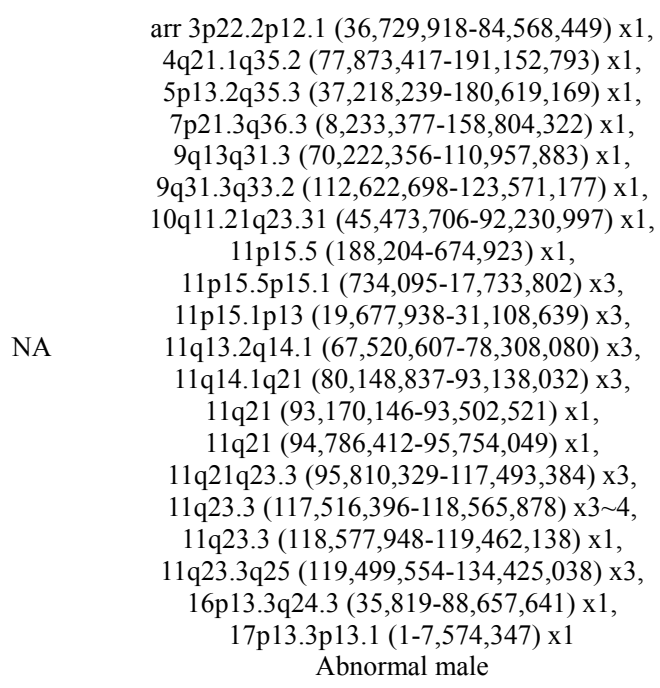

arr Xp22.33p11.21 (3,905,651-56,085,692) x2, $\begin{array}{cc}\text { nuc ish (ETV6x2, } & \text { Xq26.2q28 }(130,503,588-154,584,236) \times 2, \\ \text { RUNX1x3) } & \text { Xq28 }(154,584,237-154,876,029) \times 2,\end{array}$ RUNX1x3)
2p11.2 $(88,932,826-89,188,304) \times 1$, (ETV6 con $\quad$ 2p11.2(88,932,826-89,188,304) x1,

47, XY, + del (X) (q13q24), del (12) (p12p13) [4]/46, $\mathrm{XY},+$ del (X) (q10), del (12) (p12p13), $-13[5] / 46$, $\mathrm{XY}[11]$

46, XY, r (9) (p? 13q? 33) [12]/46, XY [18]

46, XY, t $(2 ; 12)$ (p15;q24.3), I (7) (q10),

$\mathrm{t}(9 ; 22)(\mathrm{q} 34 ; \mathrm{q} 11.2)$
25
$[9] / 47, \mathrm{sl},+8[2] / 48, \mathrm{sdl} 11$,
$+\operatorname{der}(22) \mathrm{t}(9 \cdot 22)[1] / 46$, $+\operatorname{der}(22) \mathrm{t}(9 ; 22)[1] / 46$, $\mathrm{XY}[11]$ $\begin{array}{ll}\text { [199/200], } & \text { 12p13.2p12.1 }(11,924,656-23,155,752) \times 1, \\ \text { 13q11q34 }(18,347,178-114,103,644) \times 1,\end{array}$ CEP17) $\mathrm{x} 2[500]$, (P16, ASS, ABL1,MLL, BCR) 2 [200]

ish r (9) (CDKN2A-, CEP9+, ASS-, ABL1-). nuc ish (CEP4x2, CEP10x2, D17Z1x1) [24/200], CDKN2Ax0, CEP9X2) [120/200], (ASSx1, ABL1x1, BCRx2) [153/200], MLL,ETV6, RUNX1)x2 (CEP4, CEP10, 13q11q34 (18,347,178-114,103,644) x1, $12 \mathrm{q} 21.33(90,847,497-91,060,832) \times 1$, 12p13.2p12.1 (11,924,656-23,155,752) x1,

$14 \mathrm{q} 32.33(105,402,089-106,245,460) \times 1$, $16 \mathrm{p} 11.2(30,798,095-31,840,945) \times 1$, 20 p12.2 $(10,369,721-10,470,986) \times 1$, 22q11.22 $(20,848,203-21,608,981) \times 1$ Abnormal male

arr 7p14.1 $(38,286,931-38,343,558) \times 1$, 7q34 $(141,693,456-141,719,136) \times 1$, $8 \mathrm{q} 12.1(59,952,847-60,820,059) \times 1$, $9 \mathrm{p} 24.3 \mathrm{p} 21.2(188,160-27,486,736) \times 1$, 9 p21.3 $(21,233,696-22,229,673) \times 0$, $9 p 13.2(36,965,324-36,969,492) \times 1$, 9p13.2 $(36,972,327-37,012,379) \times 3$, 9q33.2q34.3 (123,131,618-140,130,559) x1, 12q21.33 $(90,774,706-91,060,832) \times 1$, $14 \mathrm{q} 11.2(21,309,211-22,080,207) \times 1$, $14 \mathrm{q} 32.33(105,401,418-105,481,976) \times 1$ Abnormal male

Deletion of PAX5,
C20orf94, ETV6 Monosomy 13 Large gain on $\mathrm{Xp}$

$9 \mathrm{p}$ deletion with biallelic deletion of CDKN2A and $C D K N 2 B$ and terminal loss of 9qter

$P A X 5$ deletion and duplication

Deletion involving BTG1
Ig and TCRA/D deletions

FUS and MYST1 deletion arr 2p11.2 (88,932,826-89,912,416) x1, $7 \mathrm{p} 22.3 \mathrm{p} 11.1(171,273-57,515,054) \times 1$, $7 \mathrm{p} 12.2(50,381,509-50,429,675) \times 0$,

$7 \mathrm{q} 11.21 \mathrm{q} 36.3(62,030,364-158,821,424) \times 3$, 9p21.3 $(21,822,754-21,994,012) \times 1$, 9p21.3 $(21,964,305-21,994,012) \times 0$, $14 q 11.2(21,989,192-22,099,342) \times 1$, $14 \mathrm{q} 32.33(105,219,696-106,181,548) \times 1$, $20 \mathrm{p} 12.2(10,369,721-10,400,575) \times 1$, $22 \mathrm{q} 11.22(20,847,781-20,852,477) \times 1$ Abnormal male
Gain of $7 \mathrm{q} /$ loss of $7 \mathrm{p}$, consistent with $\mathrm{i}(7 \mathrm{q})$

Homozygous CDKN2A/B deletion Ig and TCR deletions

Deletions involving IKZF $1, B T G 1$ and C20orf94
Ig and TCR deletions

TOX deletion 


\section{Continued}

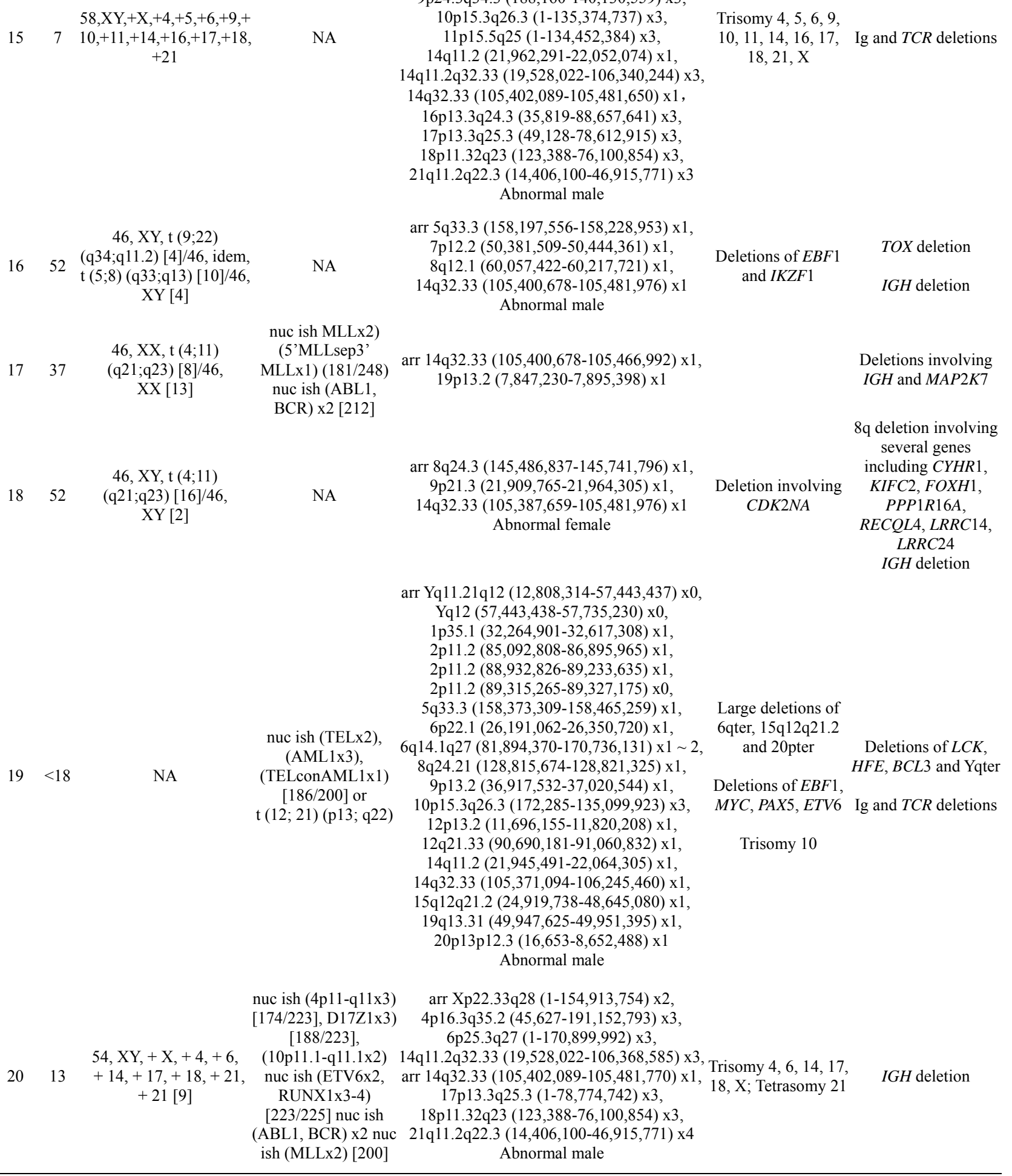
$10 \mathrm{p} 15.3 \mathrm{q} 26.3(1-135,374,737) \times 3$ $11 \mathrm{p} 15.5 \mathrm{q} 25(1-134,452,384) \times 3$,

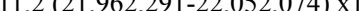
, 340,244$) \times 3$ 18p11.32q23 $(123,388-76,100,854) \times 3$ $(14,406,100-46,915,771) \times$ rr 5q33.3 $(158,197,556-158,228,953) \times 1$ $7 \mathrm{p} 12.2(50,381,509-50,444,361) \times 1$, $8 \mathrm{q} 12.1(60,057,422-60,217,721) \times 1$, $(105,400,678-105,481,976) \times$ nuc ish MLLx2) (5'MLLsep3' MLLx1) (181/248) nuc ish (ABL1, 19 p13.2 $(7,847,230-7,895,398) \times 1$

arr 8q24.3 $(145,486,837-145,741,796) \times 1$, 9 p21.3 $(21,909,765-21,964,305) \mathrm{x} 1$ 05,387,659-105,481,976) $\mathrm{x}$ ion involving CDK2NA ncluding CYHR 1 OXH P $14, L R R C 14$ LRRC24 IGH deletion

Yq11.21q12 $(12,808,314-57,443,437) \times 0$, (2) ( 33.3 $(158,373,309-158,465,259) \times 1$ $6 \mathrm{p} 22.1(26,191,062-26,350,720) \times 1$

uc ish (TELx2), (AML1x3), (TELconAML1x1) [186/200] o q24.21 $(128,815,674-128,821,325) \times 1$ . $2(36,917,532-37,020,544) \times 1$ $12 \mathrm{p} 13.2(11,696,155-11,820,208) \times 1$, $2 q 21.33(90,690,181-91,060,832) \times 1$ $14 \mathrm{q} 11.2(21,945,491-22,064,305) \times 1$, q12q21.2 $(24,919,738-48,645,080) \times 1$, 9q13.31 $(49,947,625-49,951,395) \times 1$, Abnormal male

nuc ish $(4 \mathrm{p} 11-\mathrm{q} 11 \times 3)$ 223], D17Z1x3) nuc ish (ETV6x2, RUNX1x3-4) ABL1, BCR) x2 nuc ish (MLLx2) [200]

\section{$6 \mathrm{p} 25.3 \mathrm{q} 27(1-170,899,992) \times 3$} $1.2 \mathrm{q} 32.33(19,528,022-106,368,585) \times 3$, 17p13.3q25.3 $(1-78,774,742) \times 3$, $18 \mathrm{p} 11.32 \mathrm{q} 23(123,388-76,100,854) \times 3$, Abnormal male

arr Xp22.33q28 (1-154,913,754) x2, 2 p11.2 $(89,257,482-89,277,059) \times 1$, 4p16.3q35.2 (45,627-191,152,793) x3, $5 \mathrm{p} 15.33 \mathrm{q} 35.3(129,331-180,619,169) \times 3$ 6p25.3q27 (128,203-170,736,131) x3, $7 \mathrm{p} 14.1(38,259,152-38,373,349) \times 1$, 9p24.3q34.3 (188,160-140,130,559) x3 
Table 3. Translocation microarray analysis (tCGH) for cases of B-cell ALL with known or suspected balanced translocations.

\begin{tabular}{|c|c|c|c|c|}
\hline Case & $\begin{array}{l}\text { Translocation seen by karyotype, } \\
\text { FISH and/or aCGH }\end{array}$ & Detection by tCGH & Breakpoints (hg18) & Breakpoint Distinction \\
\hline \multirow[b]{2}{*}{3} & \multirow[b]{2}{*}{$\mathrm{t}(9 ; 22)(\mathrm{q} 34 ; \mathrm{q} 11.2)$} & \multirow[b]{2}{*}{$B C R / A B L 1$} & chr9:132598080-132598270 & 5'-end $A B L 1$ \\
\hline & & & chr22:21889002-21889168 & Central m-BCR \\
\hline \multirow{2}{*}{7} & del (12) (p11.2p13), der (12) (12pter- > & \multirow{2}{*}{ ETV6/RUNX1 } & chr12:11921539-11921655 & 3'-end ETV6 \\
\hline & $\begin{array}{l}\text { 12p13::21q22->21q11.2::12p13-> 12qter), der (21) } \\
\quad(21 \text { pter- }>21 \text { q11.2::12p13::21q22- > 21qter) }\end{array}$ & & $\operatorname{chr} 21: 35330287-35331177$ & 5 '-end of intron 1 in $R U N X 1$ \\
\hline \multirow{2}{*}{8} & $\begin{array}{l}\operatorname{der}(12)(21 \text { pter- }>21 \mathrm{p} 12:: 12 \mathrm{p} 13->12 \mathrm{qter}), \text { der }(19) \\
\quad(19 \mathrm{pter}->19 \mathrm{q} 13.1:: 12 \mathrm{p} 13:: 21 \mathrm{q} 22->21 \mathrm{qqter})\end{array}$ & \multirow{2}{*}{ ETV6/RUNX1 } & chr12:11920600-11920834 & near 3'-end ETV6 \\
\hline & $\begin{array}{c}\operatorname{der}(21)(19 \mathrm{qter}->19 \mathrm{q} 13.1:: 21 \mathrm{p} 12-> \\
21 \mathrm{q} 22:: 12 \mathrm{p} 13->12 \mathrm{pter})\end{array}$ & & chr21:35204437-35204739 & 3'-end of intron 1 in $R U N X 1$ \\
\hline \multirow{2}{*}{9} & \multirow{2}{*}{$\mathrm{t}(9 ; 22)(\mathrm{q} 34 ; \mathrm{q} 11.2)$} & \multirow{2}{*}{$B C R / A B L 1$} & chr9:132582114-132583554 & 5'-end $A B L 1$ \\
\hline & & & $\operatorname{chr} 22: 21961745-21961958$ & Central M-BCR \\
\hline 10 & $\begin{array}{c}\text { Aneuploidy by karyotypes; Possible MLL gene } \\
\text { breakpoint by aCGH }\end{array}$ & None Detected & & \\
\hline \multirow{3}{*}{12} & $\begin{array}{c}\operatorname{del}(12)(\mathrm{p} 12 \mathrm{p} 13) \\
\text { nuc ish(ETV6 } \times 2, \text { RUNX1 } \times 3)\end{array}$ & \multirow{3}{*}{ ETV6/RUNX1 } & chr12:11924656-11924849 & most 3-end ETV6 \\
\hline & (ETV6 con RUNX1x1) [199/200], & & & \\
\hline & $\begin{array}{c}(\mathrm{CEP} 4, \mathrm{CEP} 10, \mathrm{CEP} 17) \times 2[500] \\
(\mathrm{P} 16, \mathrm{ASS}, \mathrm{ABL} 1, \mathrm{MLL}, \mathrm{BCR}) \times 2[200]\end{array}$ & & chr21:35266695-35266968 & center of intron 1 in $R U N X 1$ \\
\hline \multirow{2}{*}{14} & \multirow{2}{*}{$\mathrm{t}(9 ; 22)(\mathrm{q} 34 ; \mathrm{q} 11.2)$} & \multirow{2}{*}{$B C R / A B L 1$} & chr9:132635298-132635731 & Central intron 1 \\
\hline & & & chr22:21922842-21923078 & Terminal mBCR \\
\hline \multirow{2}{*}{16} & \multirow{2}{*}{$\mathrm{t}(9 ; 22)(\mathrm{q} 34 ; \mathrm{q} 11.2)$} & \multirow{2}{*}{$B C R / A B L 1$} & chr9:132651461-132651567 & Central intron 1 \\
\hline & & & chr22:21908588-21908773 & Central m-BCR \\
\hline \multirow{2}{*}{17} & \multirow{2}{*}{$\mathrm{t}(4 ; 11)(\mathrm{q} 21 ; \mathrm{q} 23)$} & \multirow{2}{*}{$M L L / A F F 1$} & chr4:88216921-88217006 & Major brpt clust $\mp$ \\
\hline & & & chr11:117860758-117860864 & Major brpt clust $\mp$ \\
\hline \multirow{2}{*}{18} & \multirow{2}{*}{$\mathrm{t}(4 ; 11)(\mathrm{q} 21 ; \mathrm{q} 23)$} & \multirow{2}{*}{$M L L / A F F 1$} & chr4:88214869-88215163 & Major brpt clust $\mp$ \\
\hline & & & chr11:117860648-117860758 & Major brpt clust $\mp$ \\
\hline 19 & $\begin{array}{c}\text { nuc ish }(\text { TEL } \times 2),(\text { AML } 1 \times 3), \\
(\text { TELconAML1 } \times 1)[186 / 200] \text { or } \mathrm{t}(12 ; 21)(\mathrm{p} 13 ; \mathrm{q} 22)\end{array}$ & $\mathrm{N} / \mathrm{D} \Phi$ & & \\
\hline
\end{tabular}

$\mathrm{TN} / \mathrm{D}=$ Not determined due to insufficient DNA; $\mp=$ Major breakpoint cluster [59].

and an additional 48.2-kb deletion within the remaining IKZF1 allele (Case 14; Table 2 and Figure 3(a)). Another case showed focal heterozygous loss of the entire IKZF1gene, but not flanking genes (Case 9; Table 2 and Figure 3(b)), and a third case had a heterozygous deletion, approximately $60 \mathrm{~kb}$ in size, involving only the 3 ' end of IKZF1 (Case 16; Table 2 and Figure 3(b)).

In addition to focal CNAs, gains and losses involving many megabases of DNA, which were only sometimes identified by conventional cytogenetic analysis, were detected by microarray (Table 2). Some of these alterations involved genes or regions previously known to be involved in ALL, such as deletions of $9 \mathrm{p}$ including CDKN2A and deletions of $12 \mathrm{p}$ including ETV6. Nine cases had losses involving $C D K N 2 A$ that ranged from intragenic deletions to deletions of many megabases. Three of these cases had heterozygous deletions ranging from $145 \mathrm{~kb}$ to 1.4 Mb (Cases 4, 9, and 18; Table 2) and six had homozygous deletions (Cases 1, 2, 5, 6, 13, and 14; Table 2 and Figure 4). In case 6 , biallelic loss of CDKN2A, including a $6.6-\mathrm{Mb}$ loss of $9 \mathrm{p} 21.3 \mathrm{p} 21.2$, was not seen by conventional cytogenetics. Of note, for case 14 deletion of one of the CDKN2A alleles that was detected by microarray was below the level of resolution of FISH $(\sim 30$ kb) (Figure 4). In Case 14, homozygous deletion of $C D K N 2 A$ was likely to be detectable only by microarray as the lesion $(\sim 30 \mathrm{~kb})$ on one allele was likely below the level of resolution of standard CDKN2A FISH probes (150 kb - $190 \mathrm{~kb})$ (Figure 4).

Losses involving ETV6 were seen in all three ETV6/RUNX1 fusion cases (Cases 7, 8, and 12; Table 2, Figure 1) and in one case that did not have a t $(12 ; 21)$ (Case 19; Table 2). Case 8 demonstrated a loss in ETV6 of $21 \mathrm{~kb}$ that was only detectable by microarray. Case 10 , which was found to have trisomies for chromosomes 6 , 13, 19 and 21 but no abnormalities of chromosome 11 by G-banded analysis, also showed multiple two-copy gains throughout $11 \mathrm{q}$ by microarray; these included a $1.2-\mathrm{Mb}$ 


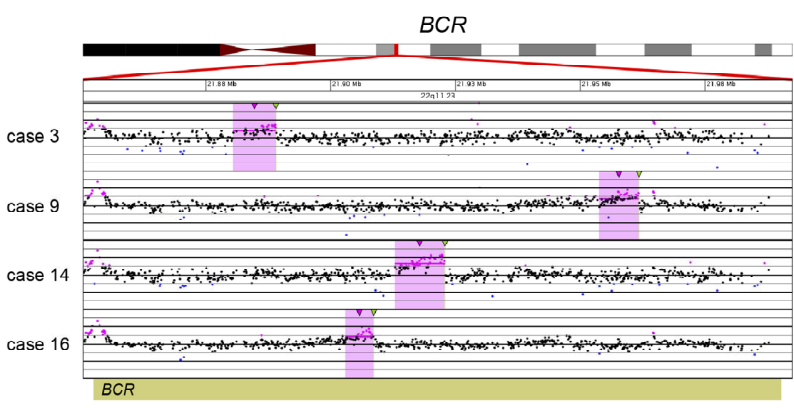

(a)

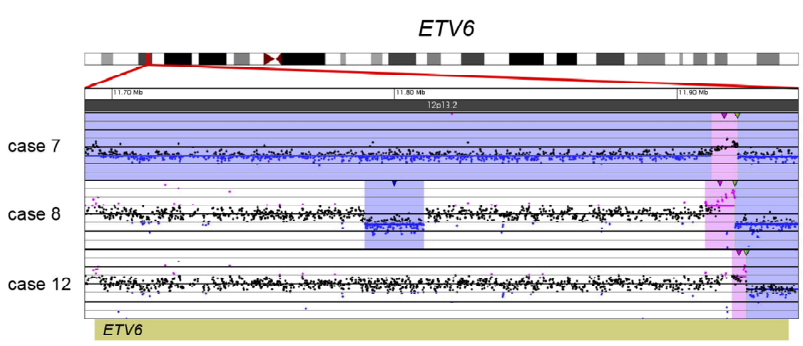

(b)

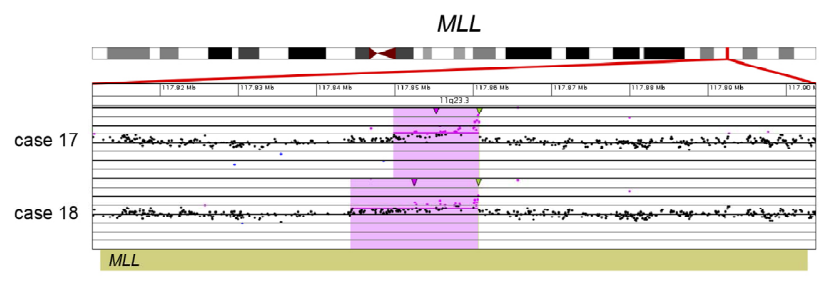

(c)

Figure 1. Balanced translocations detected by tCGH in B-cell ALL cases. The probes (pink dots) form a peak, indicating the amplification leading up to the breakpoint region (thin, green triangles). Copy gains (pink shaded regions) and copy losses (blue shaded regions) are also shown. (a) BCR/ABL1 translocations seen in cases 3, 9, 14, and 16. (b) ETV6/RUNX1 translocations seen in cases 7, 8, and 12. (c) $M L L / A F F 1$ translocations seen in cases 17 and 18. For (a)-(c), probes are ordered on the $x$-axis according to physical mapping positions, and values along the $y$-axis represent $\log _{2}$ ratios of patient:control signal intensities. Results are visualized using Oncoglyphix (Signature Genomics).

gain in 11q 23.3 with a break in the $M L L$ gene. Although one cell in this case was noted to have a marker chromosome by G-banding that could conceivably consist of 11q material, the presence of a single cell finding is not considered clonal. Even if the marker is part of the leukemic clone, the gains of 11q could not have easily been determined in this case, even by FISH. In cases that had chromosome abnormalities identified by G-banded analysis, microarray findings frequently resulted in more precise characterization of the genomic imbalances. For example, in case 11, abnormalities of chromosome 11 were noted by G-banded analysis; however, the complex pattern of chromosome 11 gains and losses detected by microarray could not be deduced from the karyotype.

Consistent with previous literature [23], cases with MLL gene rearrangements (Cases 17 and 18; Table 2) had only a few CNAs each. In contrast, cases with $t(9 ; 22)$ or $t(12 ; 21)$ frequently showed multiple CNAs. With the exception of case 3 that had a $t(9 ; 22)$ and only two CNAs, the remainder of cases with either a BCR/ABL1 (Cases 9,
14, 16, Table 2) or ETV6/RUNX1 (Cases 7, 8, and 12; Table 2) fusion had 4 to 17 CNAs.

\subsection{Detection of Hypodiploidyl Near-Haploidy}

For Case 2, the presence of four copies of many chromosomes and two of others suggested that doubling of a hypodiploid/near-haploid clone had generated a hyperdiploid pattern that can be mistaken for high hyperdiploidy [24]. This is a classic cause for concern given the negative prognostic significance of the former and positive prognostic significance of an overlapping, but distinct, hyperdiploid pattern [25]. We, therefore, analyzed Case 2 by Affymetrix SNP 6.0 microarray analysis. The SNP data showed haplotypes consistent with the doubling of a hypodiploid clone with subsequent clonal evolution allowing the poor prognostic marker to be clearly distinguished from high hyperdiploidy in this case (Figure 5). 


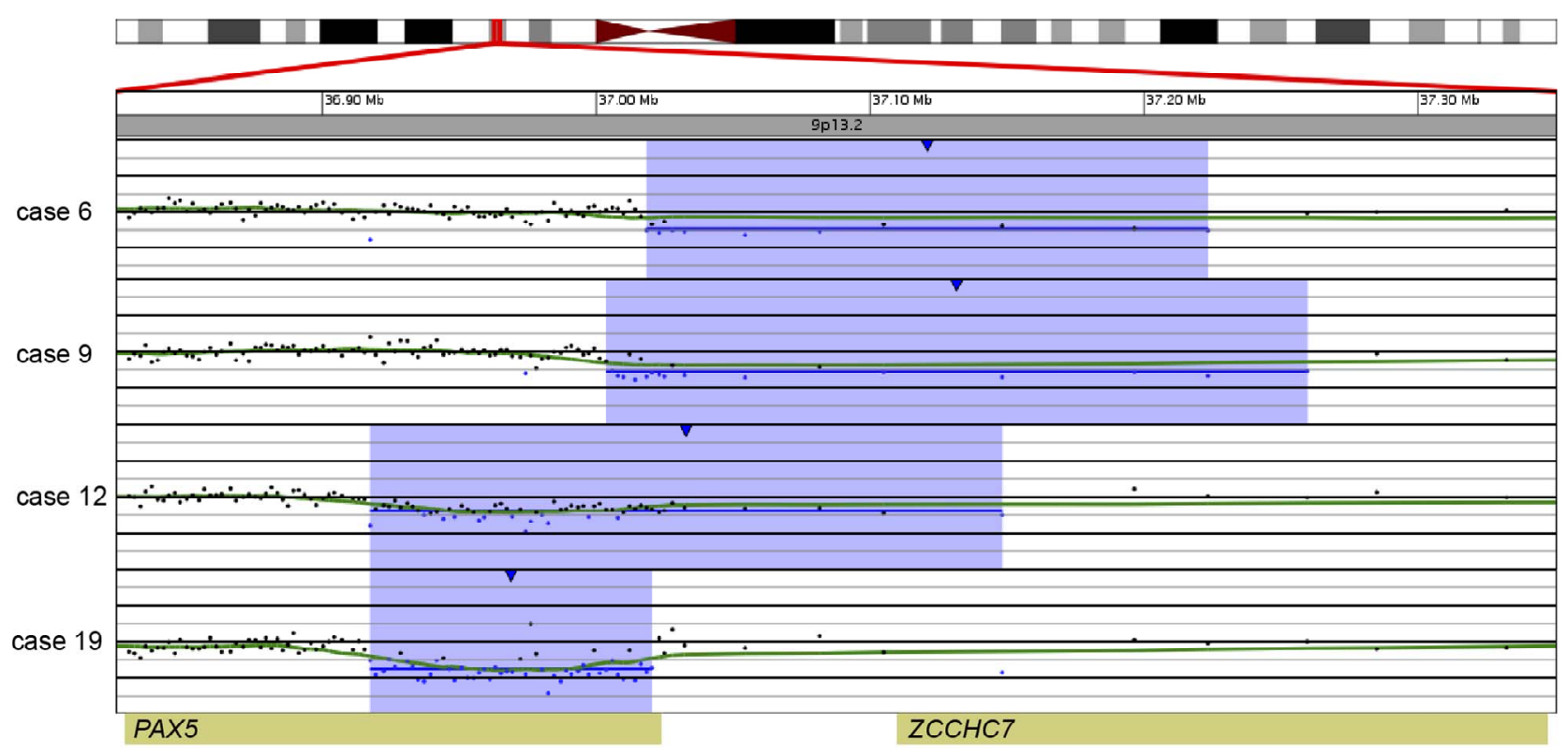

(a)

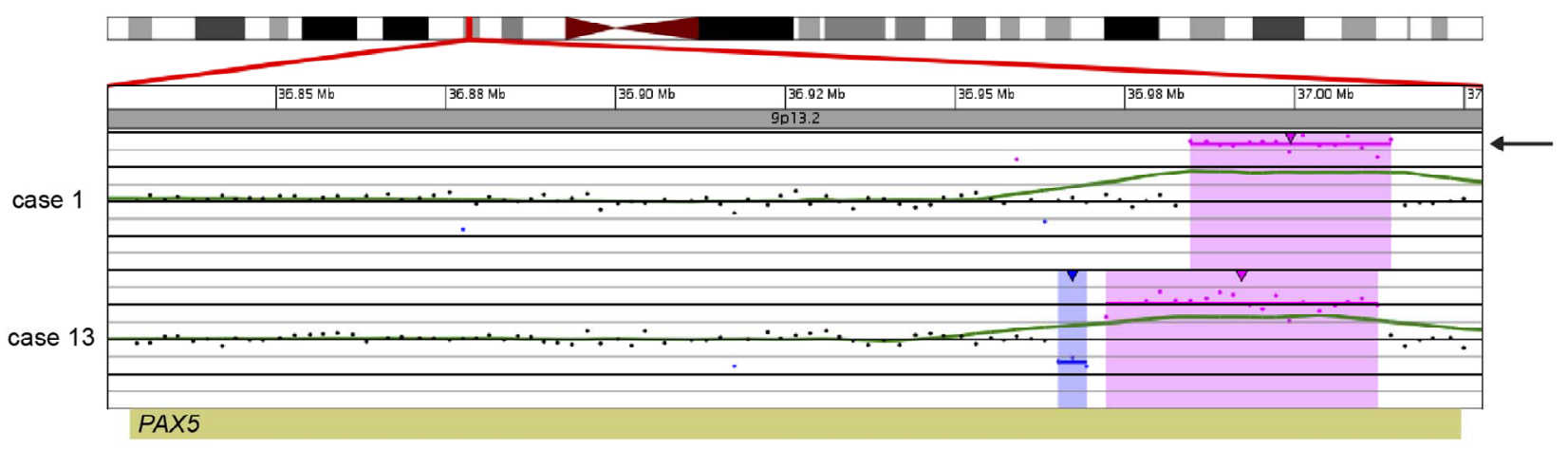

(b)

Figure 2. Copy number gains and losses of the gene $P A X 5$ at $9 \mathrm{p} 13.2$ detected by microarray analysis. $P A X 5$ is involved in the regulation of B-cell development and differentiation. (a) Cases with deletions including one or more exons at the 5' end of PAX5: Case 6 has a 205-kb loss, Case 9 has a 256-kb loss, Case 12 has a 231-kb loss, and Case 19 has a 103-kb loss. (b) Intragenic copy number alterations of PAX5: Case 1 has a two-copy gain of $30 \mathrm{~kb}$ within PAX5 (arrow) and case 13 has a 4-kb loss and a 40-kb gain within $P A X 5$. For $\mathrm{A}$ and $\mathrm{B}$, probes are ordered on the $x$-axis according to physical mapping positions, and values along the $y$-axis represent $\log _{2}$ ratios of patient:control signal intensities. Results are visualized using Oncoglyphix (Signature Genomics).

\subsection{Discrepancies between G-Banded Chromosome and Microarray Results}

Although it was expected that many CNAs detected by microarray would be missed by G-banded analysis as noted above, there were a few cases in which alterations identified by G-banded analysis could not be substantiated by the microarray results. Some cases were noted to have chromosomes with additional material of unknown origin by G-banding (Cases 1, 2, 6, 9, and 11). If these were true "add" abnormalities, they would be expected to show a deletion distal to the breakpoint of the "add" chromosome and gain of the additional chromosomal material. These imbalances were not seen by microarray. It is unlikely that these abnormalities were missed by the microarray due to low-level mosaicism, because all of the "add" chromosomes were present in a high enough proportion of cells to be detected by microarray. It is possible that some of these "add" chromosomes represent balanced rearrangements, or that they are actually normal chromosomes with distortion due to poor morphology, which is a common problem in karyotyping ALL specimens. In addition, in Case 1, deletions of $3 p$ and $9 p$ were detected by microarray that may be the result of the add ( $3 p)$ and the add (9p); however, there was no deletion of $7 q$ that would be expected from the add (7q) reported in the same clone from this case. For Case 9, a $\operatorname{del}(21)$ (q21;q22.3) was reported by G-banding, but no $21 \mathrm{q}$ deletion was detected by microarray. Again, it may be that the $21 \mathrm{q}$ abnormality consists of a balanced rear- 


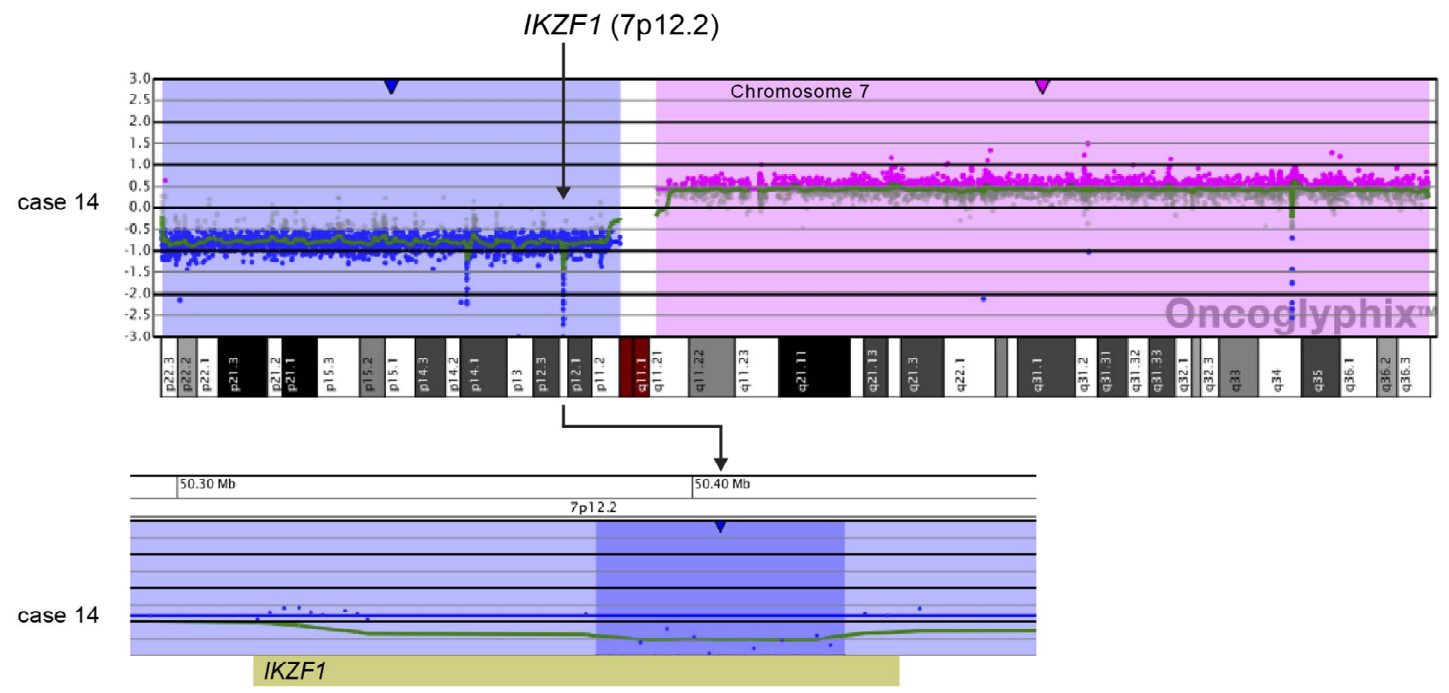

(a)

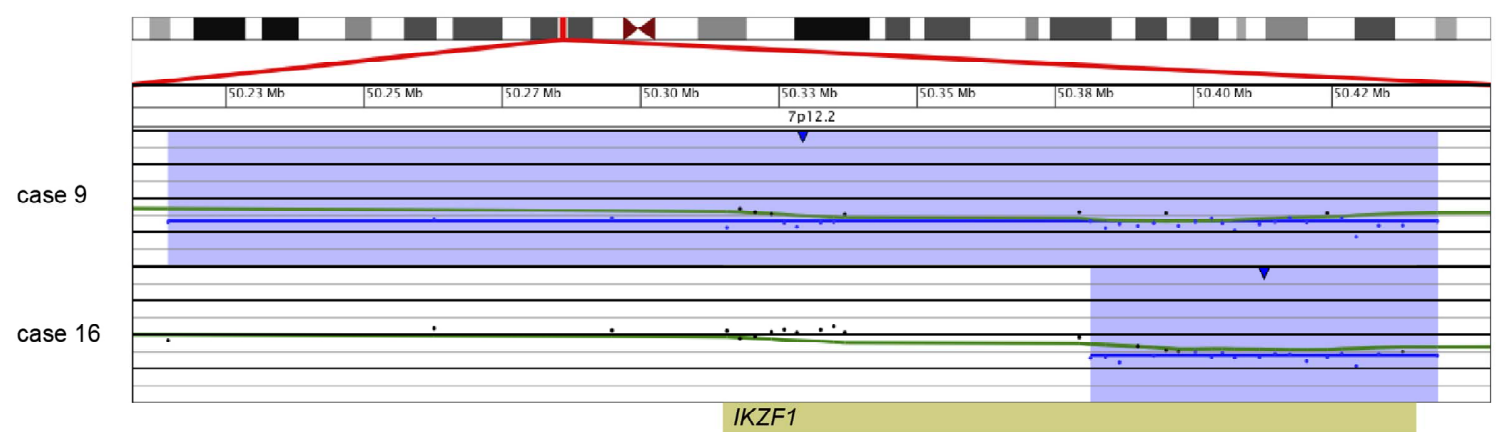

(b)

Figure 3. Copy number losses of $I K Z F 1$ at $7 \mathrm{p} 12.2$ detected by microarray analysis. $I K Z F 1$ is involved in the regulation of B-cell development and differentiation. (a) Microarray results for case 14, showing a copy number loss of the entire p-arm of chromosome 7 (shaded in blue) and a copy number gain of the entire q-arm (shaded in pink). The zoomed-in view of IKZF1 at $7 \mathrm{p} 12.2$ for case 14 shows an intragenic, biallelic $48.2 \mathrm{~kb}$ loss of part of IKZF1 (shorter arrow). (b) Microarray results for cases 9 and 16 with copy number losses (shaded in blue) of $7 \mathrm{p} 12.2$ that include IKZF1. Case 9 has a $320-\mathrm{kb}$ deletion that includes the entire gene, and case 16 has a 62-kb deletion of the 3'-end of the gene. For a and b, probes are ordered on the $x$-axis according to physical mapping positions, and values along the $y$-axis represent $\log _{2}$ ratios of patient: control signal intensities. Results are visualized using Oncoglyphix (Signature Genomics).

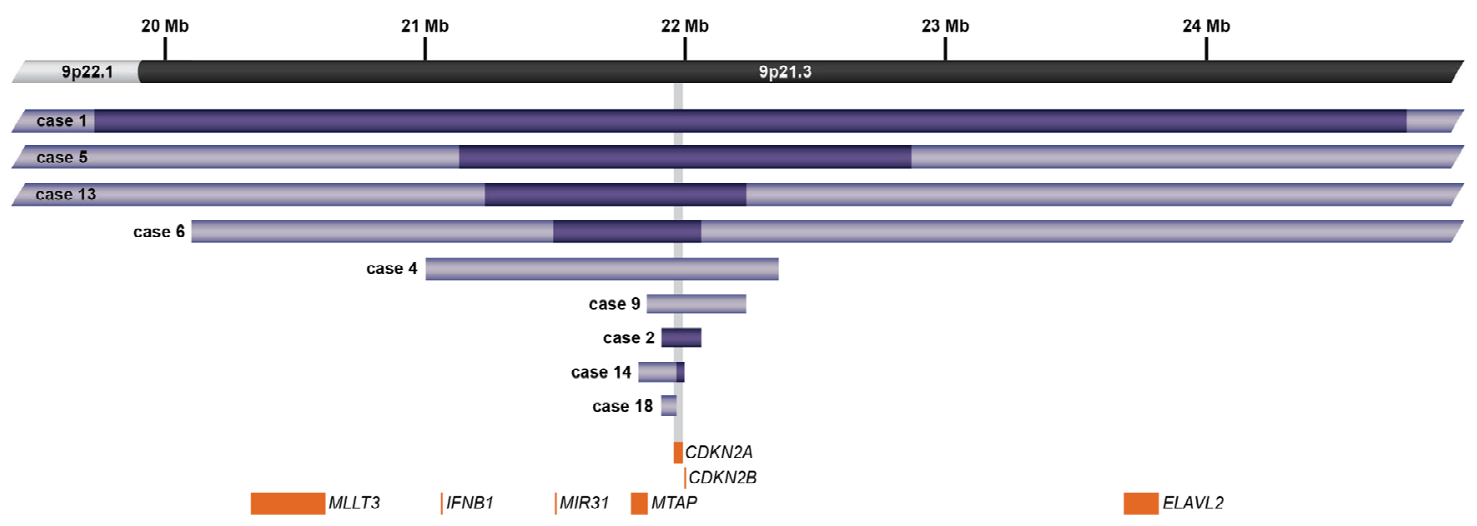

Figure 4. Schematic representation showing deletions of $C D K N 2 A$ at $9 \mathrm{p} 21.3$ detected by microarray analysis in nine cases. CDKN2A is involved in cell cycle regulation and tumor suppression. Light purple bars represent deletion sizes for each case (based on UCSC $2006 \mathrm{hg} 18$ assembly); the deletions in Cases 1, 5, 6, and 13 are larger than the genomic region shown. Cases 1, 2, 5, 6, 13, and 14 had biallelic deletions represented by dark purple bars. Orange boxes represent cancer features of interest in the region. 


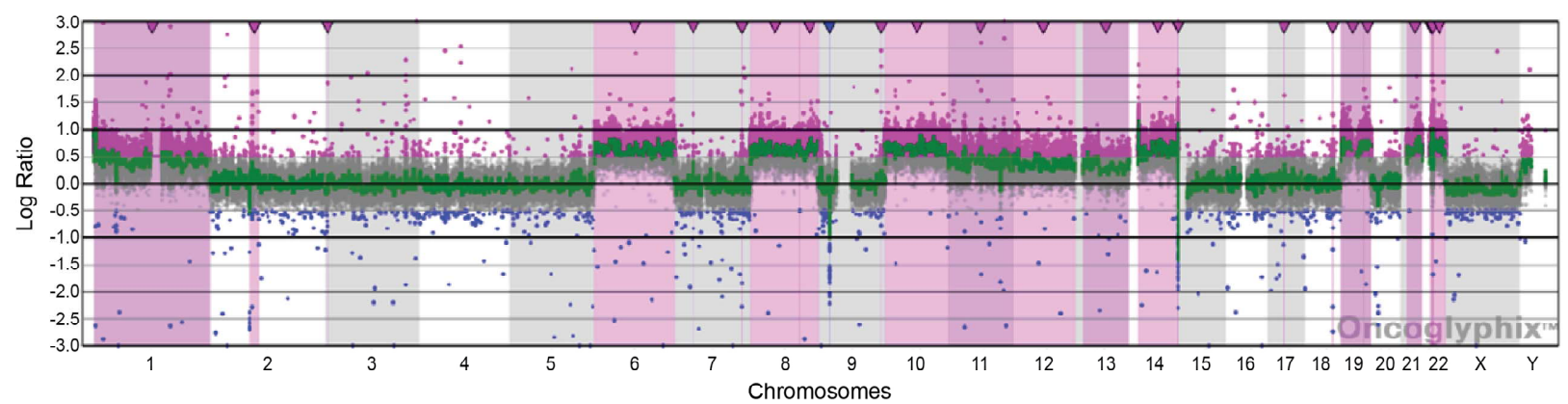

(a)

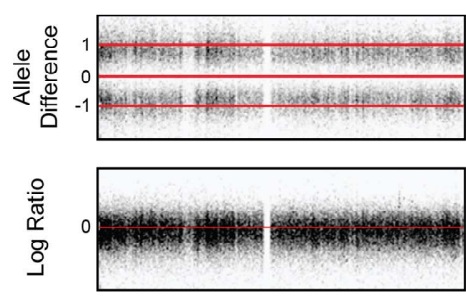

Chromosome 3

(b)
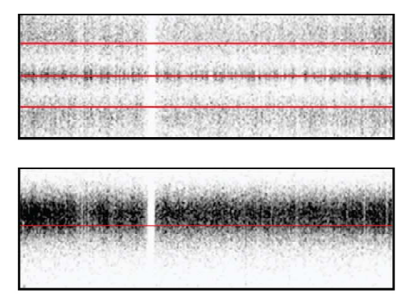

Chromosome 6

(c)
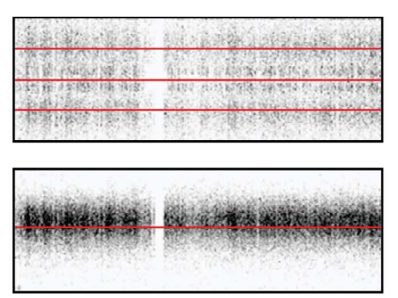

Chromosome 11

(d)

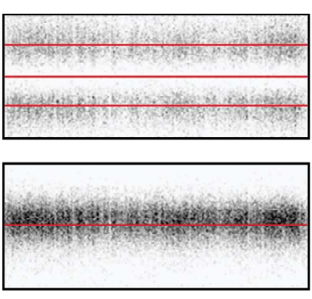

Chromosome 13

(e)

Figure 5. Copy number and SNP microarray analysis of Case 2. (a) aCGH microarray analysis of Case 2 with the Signature OncoChip. Microarray probes are ordered on the $x$-axis according to physical mapping positions, with data for chromosomes $1-22, X$, and Y being displayed from left to right, and values along the $y$-axis represent $\log _{2}$ ratios of patient: control signal intensities. Copy gains are shown as pink shaded regions. Variations in $\log _{2}$ ratios suggest different clones are present in this patient sample. The presence of four copies of many chromosomes and two of others was suggestive of doubling of a hypodiploid clone. (b)-(e) Affymetrix SNP 6.0 analysis of Case 2. The allelic difference for the SNP probes is shown at the top of each panel with the log ratio for the copy number probes shown at the bottom. (b) Data for chromosome 3 are shown as a representative example for chromosomes $\mathrm{X}, 2,3,4,5,7,9,15,16,17,18$, and 20, which showed identical patterns. These data are consistent with a copy number of 2 and loss of heterozygosity with the presence of only two genotypes (AA and BB) (c) Data for chromosome 6 are shown as a representative example for chromosomes $6,8,10,14,19,21$, and 22, which showed identical patterns. These data are consistent with a copy number of 4 and no loss of heterozygosity with the presence of only three genotypes (AAAA, AABB, and BBBB). (d) Data for chromosome 11 are shown as a representative example for chromosomes 1, 11, and 12, which showed identical patterns. These data are consistent with a copy number of three chromosomes and no loss of heterozygosity with the presence of four genotypes (AAA, AAB, BBA, and BBB). (e) Data for chromosome 13 are shown, the only chromosome in this patient sample with this pattern. These data are consistent with a copy number of three chromosomes and loss of heterozygosity with the presence of only two genotypes (AAA and BBB). The presence of homozygosity in the chromosomes with two copies as well as the presence of heterozygosity in the chromosomes with four copies is consistent with doubling of a hypodiploid clone. For (a)-(e), results are visualized using Oncoglyphix (Signature Genomics).

rangement, with $21 \mathrm{q}$ material missing from one chromosome 21 homologue but inserted elsewhere in the genome. In case 11 , monosomy 7, 9, and 10 were reported by G-banded analysis. The microarray results for this case showed loss of most of chromosome 7 but normal copy number from $7 \mathrm{p} 21.3$ to the terminus, an interstitial loss of $9 q$ but normal copy number for the remainder of chromosome 9, and an interstitial deletion of $10 \mathrm{q}$ with normal copy number for the rest of chromosome 10. As this case had a complex karyotype, it is not surprising that the retained segments of chromosomes 7, 9, and 10 could be present in a rearranged form that was not identifiable by G-banding. These cases illustrate that microarray analysis can clarify the karyotype results and provide a more accurate and unbiased assessment of imbalances in the cancer genome.

\section{DISCUSSION}

Conventional cytogenetics has been the standard of care for the identification of diagnostically and prognostically significant genomic alterations in ALL, despite the inherent challenge posed by these cases due to poor chromosome morphology. Although FISH has allowed for the detection of known alterations regardless of the presence of metaphase cells or poor morphology, the number of FISH tests that can be performed on a given case is limited, and some of the recently detected deletions of known clinical significance are below the resolution level of FISH. Despite those limitations and in spite of the relative small size of the cohort studied, the cases examined showed an age-related pattern of abnormalities highly consistent with that previously reported ([26]). 
Hyperdiploidy and ETV6/RUNX1 translocations were seen exclusively in pediatric cases, while $B C R / A B L 1$ fusions were restricted to adults.

In this study, microarray analysis allowed for the identification of CNAs below the resolution of chromosome analysis and FISH in $75 \%$ of cases. Furthermore, a novel application of linear amplification prior to aCGH, termed tCGH, identified all known translocations in nine specimens. In addition, the translocation breakpoints were identified to within a few hundred base pairs, and in some cases submicroscopic deletions at the translocation breakpoints were evident by the microarray analysis.

Most cases in our study also had additional CNAs detected by microarray, some of which are known to be prognostically significant as summarized below. Similar to other recent studies, CNAs in genes that are involved in B-cell development and differentiation were frequently detected in ALL. Other less frequent deletions in genes involved in B-cell development and differentiation that we detected have been reported in other studies of ALL and include LEF1, BTLA, and EBF1 [27].

$P A X 5$, one of the most frequent targets of alterations in ALL, encodes a transcription factor required for Blineage commitment and maturation [28]. Consistent with recent array studies [10-12], PAX5 losses or gains were present in $33 \%$ of the ALL cases tested here (Figure 3). Not including a case with loss of the entire $9 p$ due to a dicentric $(9 ; 20)$, all other cases with CNAs involving PAX5 were either intragenic or only a few hundred kilobases in size. For the current study, cases 9 and 12 showed PAX5 CNAs and were BCR/ABl1 and ETV6/ $R U N X 1$ positive, respectively, with the remaining 4 cases being translocation negative. The PAX5 CNAs consisted of both deletions and amplifications. Mutations in PAX5, including intragenic amplification as seen in case 1, have been previously described in B-cell ALL, although such alterations appear not to be correlated with a particular outcome [16,29].

Also consistent with recent literature, all cases with a t $(9 ; 22)$ in our study were found to have loss of IKZF1 [30]. In one of these cases (Case 16), the deletion was only $63 \mathrm{~kb}$ in size, which is below the resolution of FISH probes that are typically used by clinical laboratories. Deletions of IKZF1, which encodes the early lymphoid transcription factor IKAROS, have been found in $84 \%$ of $B C R / A B L 1$-positive and $28 \%$ of $B C R / A B L 1$-negative ALL $[17,30]$. IKZF1 alterations have been shown to be associated with poor outcome both in BCR/ABL1-positive and $B C R / A B L 1$-negative ALL $[13,27,31]$. Thus, the ability to detect $I K Z F 1$ deletions is critical for appropriate risk stratification.

Deletions in 8q12, ranging from $160 \mathrm{~kb}$ to $4.3 \mathrm{Mb}$, were detected by microarray in three of our cases. All three of these deletions included or partially overlapped
TOX (KIAA080). This gene has been proposed as one of two candidate genes in recurring $8 \mathrm{q} 12.1$ deletions that are present in approximately $4 \%$ of ALL [32]. TOX, originally shown to play a role in T-cell development, has recently been found to be involved in the development of many cell lineages of the immune system [33]. In Case 16, the $160-\mathrm{kb}$ deletion of $8 \mathrm{q} 12.1$ includes the first 5' exon of TOX and does not involve any other genes; therefore, our results further support TOX as a significant candidate gene within these $8 \mathrm{q} 12.1$ deletions.

The microarray results also identified deletions of genes involved in glucocorticoid resistance. Synthetic glucocorticoids are used in the treatment of pediatric ALL due to their ability to induce apoptosis in leukemic blasts; resistance to glucocorticoids is predictive of poor therapy outcome [34]. Focal deletions of BTG1 were present in four of our cases. Deletion of BTG1 is frequently seen in ALL, and loss of BTG1 expression has been shown to result in glucocorticoid resistance [35]. One case in our study demonstrated a deletion within NR3C1 (Case 6), and another had a biallelic loss within GSTT1 (Case 1). Germline mutations in NR3C1 have been found in patients with glucocorticoid resistance, and deletions of this gene have also been described in ALL [27]. Polymorphisms in the glutathione S-transferase (GST) genes have been implicated in glucocorticoid resistance. Inherited homozygous deletions of GSTT1 have been associated with an initial good response to prednisone, although the association between GST polymorphisms and relapse is controversial [36-39].

Other CNAs of interest detected in the cases studied here include frequent $C D K N 2 A$ losses $(9 / 20 ; 45 \%)$ ranging from small intragenic deletions to many megabases in size (Figure 4). The frequency of losses seen here was higher than that reported in some previous studies [40, $41]$, although our findings agreed with others $[17,42]$. Six of the CDKN2A deletions appeared homozygous by array, while the remaining cases may reflect either heterozygosity or homozygosity in a subclone of cells, given the inherent non-cellular limitations of array technology. In a previous study of childhood ALL, CDKN2A deletion was found more frequently in cases with t $(9 ; 22)(61 \%)$ than with $\mathrm{t}(12 ; 21)(15 \%)$ or $M L L$ gene rearrangements (13\%) [40]. The present study showed similar results with $C D K N 2 A$ deletion found in $2 / 4$ cases with $\mathrm{t}(9 ; 22)$, $1 / 2$ cases with a $M L L$ gene rearrangements (Case 18) and in none of the cases with $\mathrm{t}(12 ; 21)$.

Cases 17 and 18 exhibited MLL/AFF1 translocations and represent cases with the least number of CNAs detected by aCGH. This is consistent with the notion that $M L L$ gene rearrangement is a potent and sufficient oncogenic driver mutation. Of those cases exhibiting $C D K N 2 A$ deletion, case 18 exhibited deletion of CDKN $2 A$ at an apparently heterozygous level, the only clini- 
cally significant finding in addition to the $M L L / A F F 1$ translocations in the case. In contrast, all three cases with a t $(12 ; 21)$ cases showed intermediate levels of instability as measured by CNAs, including loss of or deletion in ETV6. In addition, one $\mathrm{t}(12 ; 21)$ case showed biallelic loss at the 5 '-end of TBL $1 X R 1$, which has also been found to be a recurrent event in this ALL subtype [43].

The cases showing the greatest number of CNAs were those that did not exhibit recurrent translocations, perhaps a consequence of cellular evolution in search of an oncogenic threshold (e.g., Cases 1, 6, 10, 11, and 13). Of these, Case 1 exhibited a substantial number of CNAs that included both homozygous deletion of CDKN2A and a two-copy gain within the PAX5 locus. As both abnormalities reside on chromosome $9 p$ and are homozygous, the results are suspicious for copy neutral loss of heterozygosity event, although confirmatory SNP analysis was not performed. As shown in Figure 2, the PAX5 two-copy duplication occurs within the gene. Although duplications in PAX5 have previously been thought to result in loss-of-function alleles [12], this duplication involves exons 2-5 that encode the Paired box DNA binding domain that potentially produces an in-frame product. A similar duplication involving the same specific exons was seen in case 13 (Figure 4), a case that also exhibited homozygous deletion of CDKN2A. Thus, a novel PAX5 function related to paired box duplication may be related to greater instability, homozygous CDKN2A mutation and lack of translocation, features shared by these two cases.

In the current study, copy number variation of the immunoglobulin and T-cell receptor genes were noted due to high-density coverage within these genes (see Table 2). Such alterations may also be observed in normal individuals due to somatic rearrangement; however, in hematological malignancies, these copy number changes can be indicative of clonal cell populations (see Table 2). Clonality, if present, may be related to viral infection, immunodeficiency, or neoplasia [44]. Thus, gains and losses of these regions present a unique challenge in the analysis and interpretation of oncology aCGH cases. Further studies employing additional clinical information and a standardized statistical means of analysis will be required for interpreting copy number variation in these regions and ascertaining potential clinical significance of these alterations.

It is important to note that although the aCGH analysis in Case 2 was suggestive of the doubling of a hypodiploid/near-haploid clone, the confirmation of this abnormality was only made possible by subsequent SNP microarray analysis. This information is critical to this case in order to distinguish apparent hyperdiploidy that is associated with a good prognosis from true hypodiploidy that is associated with a poor prognosis.
The tCGH technology offers unprecedented resolution of translocation breakpoints affording a new opportunity to explore the potential clinical and biological value of such data. To this end, Case 9 is of potential interest. Of the $B C R / A B L 1$ positive cases, Case 9 had the most CNAs detected by aCGH and represented newly diagnosed disease. The current data provide high-resolution analysis of the genomic breakpoints for each recurrent translocation. Relative to the other $B C R / A B L 1$ positive cases in this study, for Case 9 those breakpoints map to the furthest 3 '-end of intron 1 for $B C R$ and the furthest 5 '-end of intron 1 for $A B L 1$, yielding a genomic fusion gene larger than for other $B C R / A B L 1$ translocations. Although all four $B C R$ and $A B L 1$ breakpoints occurs within the first introns of those genes, it is interesting to speculate that regulatory and/or cryptic alternative splice sites might influence the oncogenic potential for the specific fusions. The additional genomic landscape included in the fusion for Case 9 may necessitate the acquisition of additional driver mutations (e.g., deletions of CDKN2A, PAX5, $I K Z F 1)$ to produce disease. A similar albeit less dramatic example comes from data comparison for Cases 17 and 18. Cases 17 and 18 exhibited MLL/AFF1 translocations and represent cases with the least number of CNAs detected by aCGH. This is consistent with the notion that MLL gene rearrangement is a potent and sufficient oncogengic driver. Consistent with that assumption these cases show minimal CNAs. As determined by tCGH the cases share nearly identical MLL gene breakpoints within the common cluster region (Case 17 = chr11:117860758-

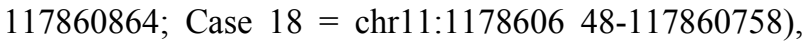
with AFF1 breakpoints separated by only a few kb (case $17=$ chr4:88216921-88217006; case $18=$ chr4:882148 69-88215163). Once again, one could speculate that the precise sequence context of the breakpoints or specific elements contained in the sequences of difference observed (several $\mathrm{kb}$ on chromosome 4) was a contributing factor for Case 18 acquiring the additional oncogenic lesion at $C D K N 2 A$. Similar proposals could be generated for the significance of ETV6/RUNX1 breakpoints. Obviously a much larger study would be required to discern to what degree breakpoints correlate with presence of additional oncogene CNAs.

The current study was completed with cases all previously analyzed by chromosome and/or FISH analysis. One potential limitation of microarray-based analysis for leukemia is that separate clones cannot be distinguished; however, current studies indicate that the presence or absence of certain CNAs at diagnosis, such as IKZF1 deletions, are important for determining relapse, regardless of whether they represent the predominant clone at diagnosis [45]. Also certain alterations may be inferred from microarray results, although the mechanism that leads to the alteration(s) may only be confirmed by con- 
ventional cytogenetic methods that allow visualization of the chromosomes. For example, microarray results on case 5 showed loss of $9 p$ and $20 q$ that would suggest the presence of a dic $(9 ; 20)$, although confirmation of this finding requires visualization of the metaphase cells by G-banding or FISH. However, if the loss of genetic material is the prognostically significant finding, which it appears to be in the case of the dic $(9 ; 20)[46,47]$, then the specific mechanism of loss is probably not as important as the genetic content of the altered region. Another issue that clinical laboratories will need to address is the potential of uncovering germline CNAs during the course of testing neoplastic specimens for acquired alterations. For example, deletions in the $D M D$ gene have been uncovered in studies of ALL [27] (see also our case 6). Other examples are given in the study performed by Dougherty et al., 2011) [3]. A non-neoplastic source of DNA from the patient may be required to determine if these are acquired or germline alterations and families would ideally receive appropriate pre-test counseling for these scenarios.

Despite the aforementioned issues, the ability to detect diagnostically and prognostically significant translocations and CNAs by microarray has significant advantages over conventional cytogenetics, with the ability to detect deletions below the resolution of FISH. Thus, arrays will be an important adjunct to conventional cytogenetics, and may eventually become the standard for first-tier testing. The ability to detect both prognostically significant balanced rearrangements and genomic imbalances is a major step toward implementing arrays in the clinical laboratory for evaluation of ALL and other forms of neoplasia.

\section{ACKNOWLEDGEMENTS}

We thank Erin Dodge (Signature Genomics) for figure creation and critical editing of the tables and manuscript and A. Michelle Caldwell (Signature Genomics) for critical editing of the manuscript.

\section{REFERENCES}

[1] Pui, C.H., Pei, D., Sandlund, J.T., Ribeiro, R.C., Rubnitz, J.E., Raimondi, S.C., et al. (2010) Long-term results of St Jude Total Therapy Studies 11, 12, 13A, 13B, and 14 for childhood acute lymphoblastic leukemia. Leukemia, 24, 371-382. doi:10.1038/leu.2009.252

[2] Pui, C.H., Campana, D., Pei, D., Bowman, W.P., Sandlund, J.T., Kaste, S.C., et al. (2009) Treating childhood acute lymphoblastic leukemia without cranial irradiation. The New England Journal of Medicine, 360, 2730-2741. doi:10.1056/NEJMoa0900386

[3] Dougherty, M.J., Wilmoth, D.M., Tooke, L.S., Shaikh, T.H., Gai, X., Hakonarson, H., et al. (2011) Implementation of high resolution single nucleotide polymorphism array analysis as a clinical test for patients with hema- tologic malignancies. Cancer Genetics, 204, 26-38. doi:10.1016/j.cancergencyto.2010.10.007

[4] Fielding, A.K., Richards, S.M., Chopra, R., Lazarus, H.M., Litzow, M.R., Buck, G., et al. (2007) Outcome of 609 adults after relapse of acute lymphoblastic leukemia (ALL); an MRC UKALL12/ECOG 2993 study. Blood, 109, 944-950. doi:10.1182/blood-2006-05-018192

[5] Nguyen, K., Devidas, M., Cheng, S.C., La, M., Raetz, E.A., Carroll, W.L., et al. (2008) Factors influencing survival after relapse from acute lymphoblastic leukemia: A Children's Oncology Group study. Leukemia, 22, 21422150. doi:10.1038/leu.2008.251

[6] Pui, C.H., Relling, M.V. and Downing, J.R. (2004) Acute lymphoblastic leukemia. The New England Journal of Medicine, 350, 1535-1548. doi:10.1056/NEJMra023001

[7] Malempati, S., Gaynon, P.S., Sather, H., La, M.K. and Stork, L.C. (2007) Outcome after relapse among children with standard-risk acute lymphoblastic leukemia: Children's Oncology Group study CCG-1952. Journal of Clinical Oncology, 25, 5800-5807. doi:10.1200/JCO.2007.10.7508

[8] Pui, C.H., Robison, L.L. and Look, A.T. (2008) Acute lymphoblastic leukaemia. Lancet, 371, 1030-1043. doi:10.1016/S0140-6736(08)60457-2

[9] Mullighan, C.G. (2011) New strategies in acute lymphoblastic leukemia: Translating advances in genomics into clinical practice. Clinical Cancer Research, 17, 396400. doi:10.1158/1078-0432.CCR-10-1203

[10] Kawamata, N., Ogawa, S., Zimmermann, M., Kato, M., Sanada, M., Hemminki, K., et al. (2008) Molecular allelokaryotyping of pediatric acute lymphoblastic leukemias by high-resolution single nucleotide polymorphism oligonucleotide genomic microarray. Blood, 111, 776-784. doi:10.1182/blood-2007-05-088310

[11] Kuiper, R.P., Schoenmakers, E.F., van Reijmersdal, S.V., Hehir-Kwa, J.Y., Van Kessel, A.G., Van Leeuwen, F.N., et al. (2007) High-resolution genomic profiling of childhood ALL reveals novel recurrent genetic lesions affecting pathways involved in lymphocyte differentiation and cell cycle progression. Leukemia, 21, 1258-1266. doi:10.1038/sj.leu.2404691

[12] Mullighan, C.G., Goorha, S., Radtke, I., Miller, C.B., Coustan-Smith, E., Dalton, J.D., et al. (2007) Genomewide analysis of genetic alterations in acute lymphoblastic leukaemia. Nature, 446, 758-764. doi:10.1038/nature 05690

[13] Kuiper, R.P., Waanders, E., Van Der Velden, V.H., Van Reijmersdal, S.V., Venkatachalam, R., Scheijen, B., et al. (2010) IKZF1 deletions predict relapse in uniformly treated pediatric precursor B-ALL. Leukemia, 24, 1258-1264. doi:10.1038/leu.2010.87

[14] White, M.K. and McCubrey, J.A. (2001) Suppression of apoptosis: Role in cell growth and neoplasia. Leukemia, 15, 1011-1021. doi:10.1038/sj.leu.2402143

[15] Russell, L.J., Capasso, M., Vater, I., Akasaka, T., Bernard, O.A., Calasanz, M.J., et al. (2009) Deregulated expression of cytokine receptor gene, CRLF2, is involved in lymphoid transformation in B-cell precursor acute lym- 
phoblastic leukemia. Blood, 114, 2688-2698.

[16] Mullighan, C.G. (2010) Genetic Alterations in High-Risk B-Progenitor Acute Lymphoblastic Leukemia, in SIOP Education Book 2010: 42nd Congress of the International Society of Paediatric Oncology, Boston, USA, October 21-24, 2010, Agarwal, B.R., et al. Ed., International Society of Paediatric Oncology, SIOP (International Society of Paediatric Oncology), Eindhoven, 77-87.

[17] Mullighan, C.G., Su, X., Zhang, J., Radtke, I., Phillips, L.A., Miller, C.B., et al. (2009) Deletion of IKZF1 and prognosis in acute lymphoblastic leukemia. The New England Journal of Medicine, 360, 470-480. doi:10.1056/NEJMoa0808253

[18] Miller, D.T., Adam, M.P., Aradhya, S., Biesecker, L.G., Brothman, A.R., Carter, N.P., et al. (2010) Consensus statement: chromosomal microarray is a first-tier clinical diagnostic test for individuals with developmental disabilities or congenital anomalies. The American Journal of Human Genetics, 86, 749-764. doi:10.1016/j.ajhg.2010.04.006

[19] Shaffer, L.G., Schultz, R.A. and Ballif, B.C. (2012) The use of new technologies in the detection of balanced translocations in hematologic disorders. Current Opinion in Genetics \& Development, 22, 264-271. doi:10.1016/j.gde.2012.01.005

[20] Greisman, H.A., Hoffman, N.G. and Yi, H.S. (2011) Rapid High-Resolution Mapping of Balanced Chromosomal Rearrangements on Tiling CGH Arrays. The Journal of Molecular Diagnostics, 13, 621-633. doi:10.1016/i.jmoldx.2011.07.005

[21] Yu, L., Slovak, M.L., Mannoor, K., Chen, C., Hunger, S.P., Carroll, A.J., et al. (2011) Microarray detection of multiple recurring submicroscopic chromosomal aberrations in pediatric T-cell acute lymphoblastic leukemia. Leukemia, 25, 1042-1046. doi:10.1038/leu.2011.33

[22] Kolquist, K.A., Schultz, R.A, Furrow, A., Brown, T.C., Han, J.Y., Campbell, L.J., et al. (2011) Microarray-based comparative genomic hybridization of cancer targets reveals novel, recurrent genetic aberrations in the myelodysplastic syndromes. Cancer Genetics, 204, 603-628. doi:10.1016/j.cancergen.2011.10.004

[23] Mullighan, C.G. and Downing, J.R. (2009) Global genomic characterization of acute lymphoblastic leukemia. Seminars in Hematology, 46, 3-15. doi:10.1053/j.seminhematol.2008.09.005

[24] Charrin, C., Thomas, X., Ffrench, M., Le, Q.H., Andrieux, J., Mozziconacci, M.J., et al. (2004) A report from the LALA-94 and LALA-SA groups on hypodiploidy with 30 to 39 chromosomes and near-triploidy: 2 possible expressions of a sole entity conferring poor prognosis in adult acute lymphoblastic leukemia (ALL). Blood, 104, 24442451. doi:10.1182/blood-2003-04-1299

[25] Moorman, A.V., Richards, S.M., Robinson, H.M., Strefford, J.C., Gibson, B.E., Kinsey, S.E., et al. (2007) Prognosis of children with acute lymphoblastic leukemia (ALL) and intrachromosomal amplification of chromosome 21 (iAMP21). Blood, 109, 2327-2330. doi:10.1182/blood-2006-08-040436

[26] Moorman, A.V. (2012) The clinical relevance of chro- mosomal and genomic abnormalities in B-cell precursor acute lymphoblastic leukaemia. Blood Reviews. 26, 123135. doi:10.1016/i.blre.2012.01.001

[27] Mullighan, C.G. and Downing, J.R. (2009) Genome-wide profiling of genetic alterations in acute lymphoblastic leukemia: Recent insights and future directions. Leukemia, 23, 1209-1218. doi:10.1038/leu.2009.18

[28] Nutt, S.L., Eberhard, D., Horcher, M., Rolink, A.G. and Busslinger, M. (2001) Pax5 determines the identity of B cells from the beginning to the end of B-lymphopoiesis. International Reviews of Immunology, 20, 65-82. doi: $10.3109 / 08830180109056723$

[29] Nebral, K., Denk, D., Attarbaschi, A., Konig, M., Mann, G., Haas, O.A., et al. (2009) Incidence and diversity of PAX5 fusion genes in childhood acute lymphoblastic leukemia. Leukemia, 23, 134-143. doi:10.1038/leu.2008.306

[30] Mullighan, C.G., Miller, C.B., Radtke, I., Phillips, L.A., Dalton, J., Ma, J., et al. (2008) BCR-ABL1 lymphoblastic leukaemia is characterized by the deletion of Ikaros. $\mathrm{Na}$ ture, 453, 110-114. doi:10.1038/nature06866

[31] Martinelli, G., Iacobucci, I., Storlazzi, C.T., Vignetti, M., Paoloni, F., Cilloni, D., et al. (2009) IKZF1 (Ikaros) deletions in BCR-ABL1-positive acute lymphoblastic leukemia are associated with short disease-free survival and high rate of cumulative incidence of relapse: A GIMEMA AL WP report. Journal of Clinical Oncology, 27, 52025207. doi:10.1200/JCO.2008.21.6408

[32] Bardet, V., Couque, N., Cattolico, L., Hetet, G., Devaux, I., Duprat, S., et al. (2002) Molecular analysis of nonrandom 8q12 deletions in acute lymphoblastic leukemia: Identification of two candidate genes. Genes Chromosomes Cancer, 33, 178-187. doi:10.1002/gcc.10014

[33] Aliahmad, P., de la Torre, B. and Kaye, J. (2010) Shared dependence on the DNA-binding factor TOX for the development of lymphoid tissue-inducer cell and NK cell lineages. Nature Immunology, 11, 945-952. doi:10.1038/ni.1930

[34] Tissing, W.J., Meijerink, J.P., den Boer, M.L. and Pieters, R. (2003) Molecular determinants of glucocorticoid sensitivity and resistance in acute lymphoblastic leukemia. Leukemia, 17, 17-25. doi:10.1038/sj.leu.2402733

[35] Van Galen, J.C., Kuiper, R.P., Van Emst, L., Levers, M., Tijchon, E., Scheijen, B., et al. (2010) BTG1 regulates glucocorticoid receptor autoinduction in acute lymphoblastic leukemia. Blood, 115, 4810-4819. doi:10.1182/blood-2009-05-223081

[36] Davies, S.M., Bhatia, S., Ross, J.A., Kiffmeyer, W.R., Gaynon, P.S., Radloff, G.A., et al. (2002) Glutathione S-transferase genotypes, genetic susceptibility, and outcome of therapy in childhood acute lymphoblastic leukemia. Blood, 100, 67-71. doi:10.1182/blood.V100.1.67

[37] Takanashi, M., Morimoto, A., Yagi, T., Kuriyama, K., Kano, G., Imamura, T., et al. (2003) Impact of glutathione S-transferase gene deletion on early relapse in childhood B-precursor acute lymphoblastic leukemia. Haematologica, 88, 1238-1244.

[38] Meissner, B., Stanulla, M., Ludwig, W.D., Harbott, J., 
Moricke, A., Welte, K., et al. (2004) The GSTT1 deletion polymorphism is associated with initial response to glucocorticoids in childhood acute lymphoblastic leukemia. Leukemia, 18, 1920-1923. doi:10.1038/sj.leu.2403521

[39] Sulong, S., Moorman, A.V., Irving, J.A., Strefford, J.C., Konn, Z.J., Case, M.C., et al. (2009) A comprehensive analysis of the CDKN2A gene in childhood acute lymphoblastic leukemia reveals genomic deletion, copy number neutral loss of heterozygosity, and association with specific cytogenetic subgroups. Blood, 113, 100-107. doi:10.1182/blood-2008-07-166801

[40] Bertin, R., Acquaviva, C., Mirebeau, D., Guidal-Giroux, C., Vilmer, E. and Cave, H. (2003) CDKN2A, CDKN2B, and MTAP gene dosage permits precise characterization of mono- and bi-allelic 9p21 deletions in childhood acute lymphoblastic leukemia. Genes Chromosomes Cancer, 37, 44-57. doi:10.1002/gcc. 10188

[41] Usvasalo, A., Savola, S., Raty, R., Vettenranta, K., HarilaSaari, A., Koistinen, P. et al. (2008) CDKN2A deletions in acute lymphoblastic leukemia of adolescents and young adults: An array CGH study. Leukemia Research, 32, 1228-1235. doi:10.1016/j.leukres.2008.01.014

[42] Parker, H., An, Q., Barber, K., Case, M., Davies, T., Konn, Z. et al. (2008) The complex genomic profile of ETV6RUNX1 positive acute lymphoblastic leukemia highlights a recurrent deletion of TBL1XR1. Genes Chromosomes Cancer, 47, 1118-1125. doi:10.1002/gcc.20613

[43] Hodges, E., Krishna, M.T., Pickard, C. and Smith, J.L. (2003) Diagnostic role of tests for T cell receptor (TCR) genes. Journal of Clinical Pathology, 56, 1-11. doi:10.1136/jep.56.1.1

[44] Roberts, K.G. and Mullighan, C.G. (2011) How new advances in genetic analysis are influencing the understanding and treatment of childhood acute leukemia. Current Opinion in Pediatrics, 23, 34-40. doi:10.1097/MOP.0b013e3283426260

[45] Schoumans, J., Johansson, B., Corcoran, M., Kuchinskaya, E., Golovleva, I., Grander, D., et al. (2006) Characterisation of $\operatorname{dic}(9 ; 20)(\mathrm{p} 11-13 ; \mathrm{q} 11)$ in childhood B-cell precursor acute lymphoblastic leukaemia by tiling resolution array-based comparative genomic hybridisation reveals clustered breakpoints at 9p13.2 and 20q11.2. British Journal of Haematology, 135, 492-499. doi:10.1111/j.1365-2141.2006.06328.x

[46] Strefford, J.C., Worley, H., Barber, K., Wright, S., Stewart, A.R., Robinson, H.M. et al. (2007) Genome complexity in acute lymphoblastic leukemia is revealed by array-based comparative genomic hybridization. Oncogenomics, 26, 4306-4318. doi:10.1038/sj.onc. 1210190

[47] Dewald, G.W., Ketterling, R.P., Wyatt, W.A. and Stupca, P.J. (2002) Cytogenetics studies in neoplastic hematologic disorders, in clinical laboratory medicine. In: McClatchey, K.D., Ed., Lippincott Williams \& Wilkins, Philadelphia, 658-685.

[48] Pui, C.H., Frankel, L.S., Carroll, A.J., Raimondi, S.C.,
Shuster, J.J., Head, D.R. et al. (1991) Clinical characteristics and treatment outcome of childhood acute lymphoblastic leukemia with the $t(4 ; 11)(\mathrm{q} 21 ; \mathrm{q} 23)$ : A collaborative study of 40 cases. Blood, 77, 440-447.

[49] Grimwade, D., Walker, H., Oliver, F., Wheatley, K., Harrison, C., Harrison, G., et al. (1998) The importance of diagnostic cytogenetics on outcome in AML: Analysis of 1,612 patients entered into the MRC AML 10 trial. The Medical Research Council Adult and Children's Leukaemia Working Parties. Blood, 92, 2322-2333.

[50] Raimondi, S.C., Chang, M.N., Ravindranath, Y., Behm, F.G., Gresik, M.V., Steuber, C.P., et al. (1999) Chromosomal abnormalities in 478 children with acute myeloid leukemia: Clinical characteristics and treatment outcome in a cooperative pediatric oncology group study-POG 8821. Blood, 94, 3707-3716.

[51] Balgobind, B.V., Raimondi, S.C., Harbott, J., Zimmermann, M., Alonzo, T.A., Auvrignon, A., et al. (2009) Novel prognostic subgroups in childhood 11q23/MLLrearranged acute myeloid leukemia: Results of an international retrospective study. Blood, 114, 2489-2496. doi:10.1182/blood-2009-04-215152

[52] Johansson, B., Moorman, A.V., Haas, O.A., Watmore, A.E., Cheung, K.L., Swanton, S., et al. (1998) Hematologic malignancies with $\mathrm{t}(4 ; 11)(\mathrm{q} 21 ; \mathrm{q} 23)$ - A cytogenetic, morphologic, immunophenotypic and clinical study of 183 cases. European 11q23 Workshop participants. Leukemia, 12, 779-787. doi:10.1038/sj.leu.2401012

[53] Burmeister, T., Meyer, C., Schwartz, S., Hofmann, J., Molkentin, M., Kowarz, E., et al. (2009) The MLL recombinome of adult CD10-negative B-cell precursor acute lymphoblastic leukemia: Results from the GMALL study group. Blood, 113, 4011-4015. doi:10.1182/blood-2008-10-183483

[54] Sandoval, C., Head, D.R., Mirro, J.Jr., Behm, F.G., Ayers, G.D. and Raimondi, S.C. (1992) Translocation t(9;11) (p21;q23) in pediatric de novo and secondary acute myeloblastic leukemia. Leukemia, 6, 513-519.

[55] Hunger, S.P. (1996) Chromosomal translocations involveing the E2A gene in acute lymphoblastic leukemia: Clinical features and molecular pathogenesis. Blood, 87, 12111224.

[56] Raimondi, S.C., Privitera, E., Williams, D.L., Look, A.T., Behm, F., Rivera, G.K., et al. (1991) New recurring chromosomal translocations in childhood acute lymphoblastic leukemia. Blood, 77, 2016-2022.

[57] Rubnitz, J.E., Behm, F.G., Curcio-Brint, A.M., Pinheiro, R.P., Carroll, A.J., Raimondi, S.C., et al. (1996) Molecular analysis of $\mathrm{t}(11 ; 19)$ breakpoints in childhood acute leukemias. Blood, 87, 4804-4808.

[58] Meyer, C., Schneider, B., Jakob, S., Strehl, S., Attarbaschi, A., Schnittger, S., et al. (2006) The MLL recombinome of acute leukemias. Leukemia, 20, 777-784. doi:10.1038/sj.leu.2404150 